\title{
Information dynamics in neuronal networks of macaque cerebral cortex reflect cognitive state and behavior.
}

\author{
Thomas F. Varley ${ }^{1,2}$, Olaf Sporns ${ }^{1}$, Hansjörg Scherberger ${ }^{3,4}$, and Benjamin Dann ${ }^{3}$ \\ ${ }^{1}$ Department of Psychological \&3 Brain Sciences, Indiana University, Bloomington, IN, USA \\ ${ }^{2}$ School of Informatics, Computing, and Engineering, Indiana University, Bloomington, IN, USA \\ ${ }^{3}$ Neurobiology Laboratory, German Primate Center, Goettingen, Germany \\ ${ }^{4}$ Faculty of Biology and Psychology, University of Goettingen, Germany
}

September 5, 2021

\begin{abstract}
The brain is often described as "processing" information: somehow, the decentralized interactions of billions of neurons collectively are somehow able to give rise to "emergent" behaviors, such as perception, cognition, and action. In neuroscience and cognitive science, however, "information processing" is often vaguely defined, making an exact model connecting neurodynamics, information processing, and behavior difficult to pin down. While considerable previous work has examined the structure of information dynamics in cultures or models, it remains uncertain what insights information dynamics can provide about cognition and behavior in order to interact with the environment. In this paper, we use information theory and the theory of information dynamics as a formal framework to explore information processing in multi area neuronal networks recorded from three macaques engaged in sensory-motor transformations: perceiving a visual cue, preparation of a grasping movement, and movement execution. We found that different states and grasp conditions are associated with significant re-configurations of the effective network structure and the overall information flowing through the system. Crucially, differences between cognitive and behavioral states and conditions were related to changes to higher-order, synergistic information dynamics not localizable to a single pair of source/target neurons. Our results suggest that the combined application of information-theoretic analysis of dynamics and network science inference to networks of neurons is a powerful tool to probe the neuronal basis of cognition and behavior. Keywords: Information Theory, Macaque, Synergy, Transfer Entropy, Networks.
\end{abstract}

\section{Introduction}

Animal nervous systems are often described as "information processing engines:" organisms take in information about the world around them through sensory organs, learn statistical regularities in the incominginformation, and use those to navigate and interact with their environment. The nervous system itself appears to be structured in a manner effective for information-processing [1], indicating that natural selection has optimized neural function and organization in accordance with information-theoretic considerations. In the context of neural networks, we operationalize "information processing" as a decentralized process: the global next state of the system is an emergent effect of the interactions between the system structure, the 
previous internal state and the sensory input from the environment. This process has been referred to as "distributed computation" [2,3] and, in context of the brain, is thought to be a basis for cognition and behavior. Distributed computation can be understood by dividing the "information dynamics" into three parts [3]: information storage (the degree to which the past activity of a neuron informs on it's future, e.g. LTP or LTD) [4], information transfer (the degree to which the past of a source neuron informs a target neuron's future, e.g. synaptic communication) [5, 6], and information modification (i.e., "non-linear" computation where a neuron integrates distinct streams of information into something greater than the sum of it's parts) $[7,8,9,10]$. These three dynamics can be formalized using information theory [11] (see Sec. 1.1).

Previous work using information theory to study information dynamics in recorded neuronal networks found that the ability to modify information changes during development [10], and in the same developmental windows, particular patterns of information transfer are "locked-in" [12]. Furthermore, the capacity for information modification is heterogeneously distributed across neurons of the network; concentrated in highdegree, rich-club neurons [13, 14, 15]. Information transfer [16] has been applied to a variety of neural and neuronal recordings (see [5] for a comprehensive review) and allows researchers to estimate effective network models of interacting neurons. Finally, active information storage has provided insights into stimulus response and preferences in visual processing systems [4].

Despite the wealth of analyses that have been done in this field, the question of how information dynamics relate to behavior remains unclear, as much of the above-cited work has been done in neural cultures as opposed to behaving organisms interacting with a complex environment. Consequently, proposing a link between information dynamics and behaviour (e.g. what purpose a particular dynamic serves) remains difficult. For example, while the presence of synergistic information dynamics is well documented, it remains unclear what (if any) role they play for cognition and behaviour-related information processing, or if they are merely statistical epiphenomena. To this end, we examined the information dynamics and the resulting effective network structures of simultaneously recorded neural populations from up to three cortical areas of the fronto-parietal grasping network of three macaque monkeys. During recordings monkeys performed a delayed sensory-motor transformation task involving processing of different visual cues, preparation and memorization of two different grasp types, and executions of these grasp types. (for details, see [17]. With these data, we can estimate the neuron-level active information storage, information transfer, and synergistic information modification in different cognitive and behavioral states, allowing us to directly assess the relationship between information dynamics and complex behaviors. Furthermore, by inferring transfer entropy networks, we can apply techniques from network sciences $[18,19]$ to examine how changes in behavior alter the patterns of effective connectivity between neurons in the network. Finally, we can combine these two lines of analysis to explore how a neurons location in the network couples with the particular task to reveal the local role of individual neurons in information processing.

We hypothesized that different behavioral states and grip-movements would be associated with distinct patterns of information dynamics. Specifically, we hypothesized that behavioral states that require a high degree of active processing (e.g. recognizing a behavioral cue, preparing and executing an action) would display more complex activity and distinct network structure compared to other states (such as expectant fixation). Our findings were consistent with these hypotheses: different behavioral states were associated with significant re-configurations of the global effective network structure, with movement in particular being associated with an increase in the overall amount of information flowing through the system and an increase in the amount of synergistic information processing. These network-wide patterns of activity were different for each of the two different grasping types and the two behaviors could be distinguished based on the 
particular patterns of information storage and transmission. Finally, we found that a neuron's particular location in the network did provide insights into its particular computational role.

\section{$1.1 \quad$ Basic Theory of Information Dynamics}

A natural mathematical framework for assessing these computational dynamics is information theory [11, 3], which describes how the activity of interacting elements of a complex system constrain the space of possible states of the whole system. A significant advantage of information is that, being based only on marginal, joint, and conditional probabilities of events, it is both model-free and sensitive to non-linear relationships between interacting elements [20]. Information theoretic analysis is then "epistemically modest", as it does not require presupposing particular generating functions or relationships. This makes it ideal for complex, nonlinear systems, such as networks of neurons of brains, where the underlying generative dynamic is unknown and nonlinearities can play a key role [21].

Information theory quantifies the how knowledge of a variable, or set of interacting variables, reduces the uncertainty of an observer watching the system. The central object of study is the entropy of a random variable, which quantifies an observer's uncertainty about the next state of a variable under study. For a discrete random variable $X$, with support set $\mathcal{X}$, the entropy $H(X)$ is canonically given as:

$$
H(X)=-\sum_{x \in \mathcal{X}} P(X=x) \log (P(X=x))
$$

When measured in bits, the entropy of $X$ quantifies the expected number of yes/no questions required to completely specify the state of $X$ with total certainty. Given two variables $X$ and $Y$, we can calculate the mutual information between the two as the degree to which knowing the state of one variable reduces our uncertainty about the state of the other. Formally:

$$
I(X ; Y)=H(X)-H(X \mid Y)
$$

Where $H(X \mid Y)$ is the conditional entropy of $X$ given $Y$. The difference between our initial uncertainty about $X$ and the remaining uncertainty after accounting for $Y$ is the amount of uncertainty about $X$ that $Y$ resolves.

For temporally extended processes, we can break down the total information structure of the system in three information dynamics: active information storage, information transfer, and information modification. Information storage quantifies the degree to which knowing the past of a variable, or set of variables, decreases our uncertainty about its immediate future:

$$
A I S(X)=I\left(X_{0: t-1} ; X_{t}\right)
$$

Where $X_{0: t-1}$ is the joint state of all past states of variable $X$ and $X_{t}$ is the immediate next state. In the context of neurons, an example hyperpolarizing a neuron will reduce the probability that it will fire in the immediate future [4].

For sets of interacting variables, we can generalize the active information storage to quantify how much information is "transferred" from a source neuron to a target neuron by determining how much knowing the past of a prospective "source" variable $X$ reduces our uncertainty about the future of a "target" variable $Y$ 
(above and beyond the information stored in the target):

$$
T E(X \rightarrow Y)=I\left(X_{0: t-1} ; Y_{t} \mid Y_{0: t-1}\right)
$$

$T E(X \rightarrow Y)$ is referred to as the transfer entropy from $X$ to $Y$. The canonical example here is that of synaptic communication: knowing that an excitatory pre-synaptic neuron spiked increases our certainty that the post-synaptic neuron will spike in the near future. Importantly, the I/O functions of neurons themselves are highly complex [22], and so the future behavior of the neuron may depend on the collective behaviour of all the upstream sources, which can be quantified with the multivariate transfer entropy:

$$
m T E(\mathbf{Z} \rightarrow Y)=I\left(\mathbf{Z}_{0: t-1} ; Y_{t} \mid Y_{0: t-1}\right)
$$

where $\mathbf{Z}$ is an ensemble of neurons. To recover the effect of a single source on the target in the context of all other informative sources, we use the conditional multivariate transfer entropy [23, 24, 25]:

$$
m T E(X \rightarrow Y \mid \mathbf{Z})=I\left(X_{0: t-1} ; Y_{t} \mid Y_{0: t-1}, \mathbf{Z}_{0: t-1}\right)
$$

Finally, the third information dynamic, information modification, refers to information produced when multiple incoming "streams" intersect and are non-trivially changed. This is operationalized as the "synergy" $[7,26]$ (for mathematical details see Sec. 5.9). Briefly, synergy can be understood as the information provided by a set of sources that cannot be extracted from any simpler combination. While the existence of synergy in biological neural networks is extremely well verified [10,13, 14, 15, 27], its exact biological or behavioral significance remains unclear.

\section{Results}

\subsection{Behavioral task and single neuron recordings}

To study whether different behavioral states and grip-movements are associated with distinct patterns of information dynamics, we utilize the recorded data from three monkeys ( $\mathrm{S}, \mathrm{Z}$, and $\mathrm{M}$ ). Monkeys were trained to perform a delayed grasping task (Fig. 1 A). The task was divided into four epochs: (1) an initial fixation epoch, which was the same for all conditions, (2) a cue epoch, in which the monkeys were either instructed or free to choose to grasp a target with one of two possible grip types (power or precision grip; monkey $\mathrm{M}$ was only trained to performed the instructed context), (3) a memory epoch, in which the monkeys had to prepare and remember the corresponding grip-type, and a movement epoch, in which the monkeys performed the corresponding grip-type (for details, see Materials \& Methods). While monkeys performed the task, we simultaneously recorded large populations of well isolated neurons from the ventral premotor cortex (area F5), the anterior intraparietal area (AIP), and for one monkey from the hand area of the primary motor cortex (M1) (Fig. 1 B). For this purpose, two 32-channel microelectrode arrays were chronically implanted per area resulting in 128 recorded channels for monkey $\mathrm{S}$ and $\mathrm{Z}$ and 192 recording channels for monkey M. For all following analyses, we used 4 recording sessions from monkey $\mathrm{S}$ with on average $82.8+/-5.7 \mathrm{SD}$ neurons, 3 sessions from monkey $\mathrm{Z}$ with on average $53+/-5.6 \mathrm{SD}$ neurons, and 3 sessions from monkey $\mathrm{M}$ with on average $134.3+/-23.7 \mathrm{SD}$ neurons. 


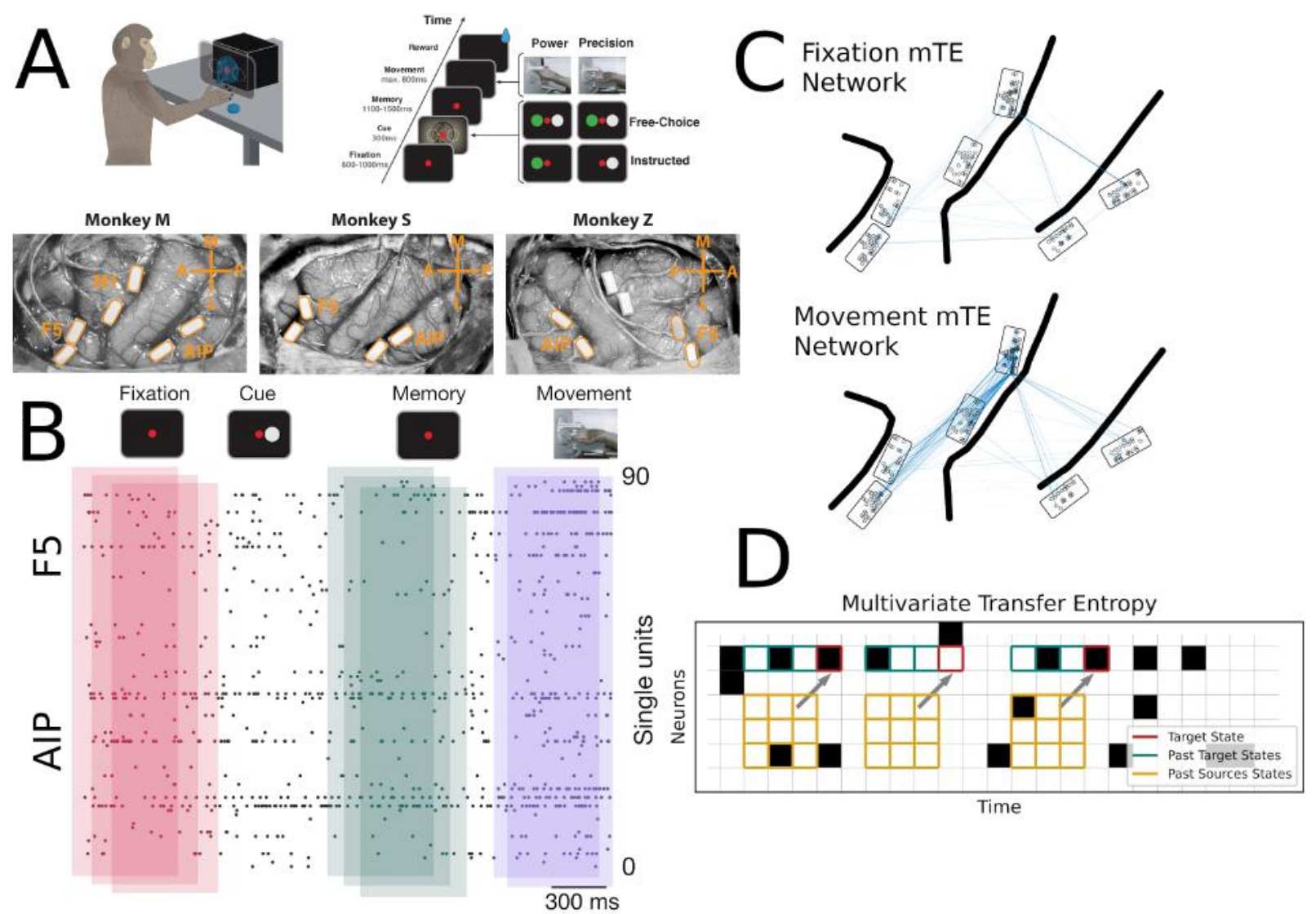

Figure 1: Electrode array implantation and behavioral task structure. A The macaque was placed in front of a screen on which distinct symbols can be displayed. The macaque had been trained that, depending on the symbol that appeared, it would execute one of two distinct grip types (Grip Type A or Grip Type B). The structure of the task was that each trial would begin with a epoch of passively fixating a red disc on a screen, followed by the onset of the cue, an epoch of return to the fixation where the appropriate movement was prepared and memorized, and finally the signal to execute the appropriate grip type. B The arrays implanted in each of the three macaque brains - note that the coverage is of three regions related to different phases of sensory-motor processing: M1, AIP, and F5. C A cartoon explaining the intuition behind the multivriate transfer entropy. We should note that the actual transfer entropy algorithm takes a continuous "sliding windows" approach - in this cartoon, only a subset of "windows" are visualized for the purposes of building intuition. D A cartoon of the sliding window structure: overlapping slices of the multivariate timeseries were used to construct a sequence of mTE networks (as well as calcuate AIS and synergy), on which we could do analysis. E visualizations of the effective networks, projected into the anatomical space for two different behavioral states (fixation and movement). 

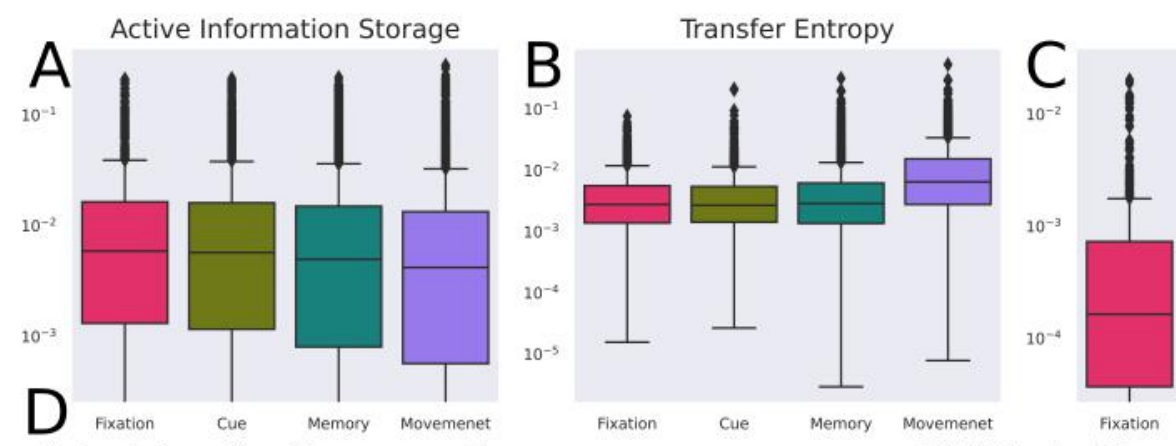

Synergy
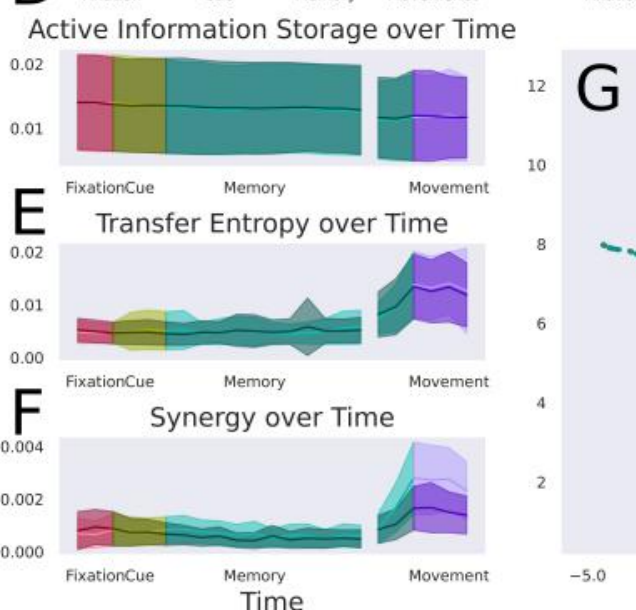

UMAP Embedding
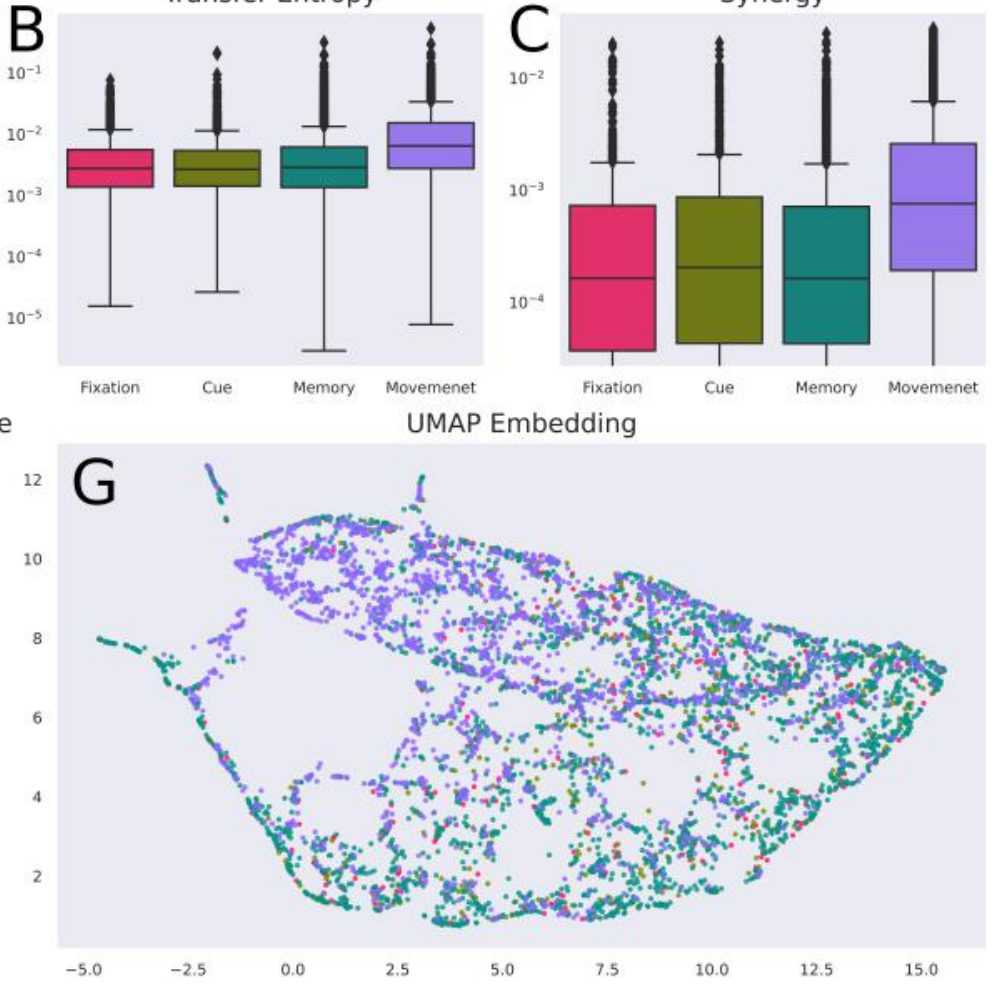

Redundency / Synergy Bias

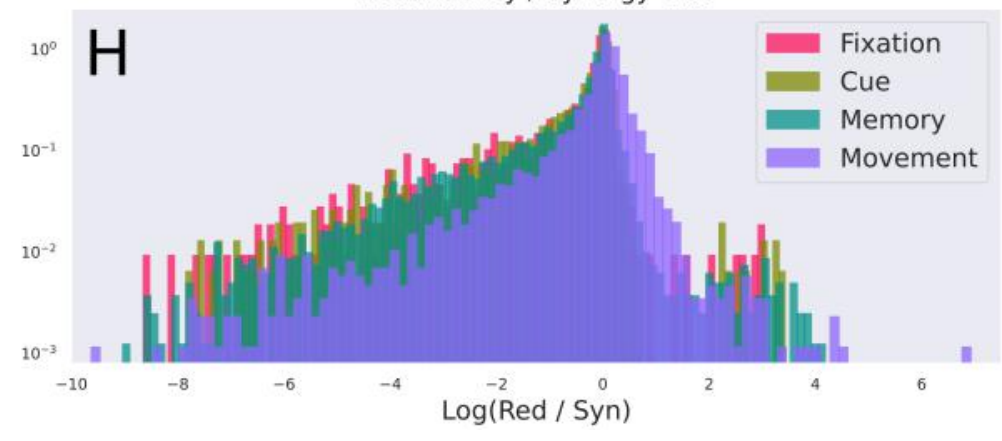

Proportion of Joint MI by PI Atom

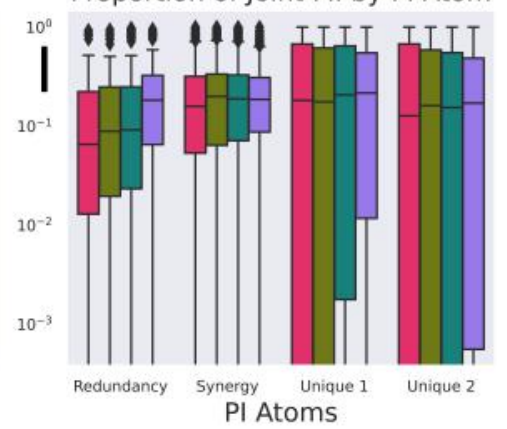

Figure 2: Information dynamics \& behavioral states: A - C. The differences in AIS (left), mTE (middle), and synergy (right) between the four behavioral states. There is no significant difference for AIS over time, however mTE and synergy show dramatic increases during the movement epoch. D - F Sliding windowed, time-resolved differences between conditions. These figures provide a more finely time-resolved picture of how information dynamics vary between behavioral states and grasp conditions. For example, we can see that the mTE and synergy were starting to increase prior to the visible onset of movement. G A UMAP [28] embedding of every node across all time. This forms a low-dimensional "dynamical morphospace" $[29,30]$ that captures the difference between distinct dynamical regimes as different regions in a configuration space. Note that the axes are unit-less. H The synergy / redundancy bias between behavioral states plotted as histograms. All the states are, on average, synergy-biased, although the onset of the movement behavior is associated with a distinct increase in the proportion of information that is redundant. I The distribution of partial-information atoms across all triads, normalized by the proportion of the total joint mutual information accounted for in each triad. Interestingly, in this framework, a dramatic increase in redundancy during movement becomes apparent. 


\subsection{Information Dynamics}

To examine behavior dependent changes in information dynamics, we estimated AIS, mTE and synergy between all simultaneously recorded neurons (see Materials \& Methods). All three measures were computed over the time course of the task with a sliding window of $800 \mathrm{~ms}$ and separate per grasping condition (step size of 100ms, 23 time windows x 2 grasping conditions; (Fig. $1 \mathrm{C}$ ). Note that in order to reduce the influence of behavior dependent firing rate changes, the AIS and the mTE measures were significance tested with conservatively estimated surrogate data (shuffling spikes within $25 \mathrm{~ms}$ windows and recomputing the corresponding measure; for details, see Materials \& Methods). In addition, all values (AIS, mTE, and synergy) were normalized by dividing by the Shannon entropy of the receiver neuron, following [13, 14, 15], which provides a control for variable firing rates. For a first assessment of differences in information dynamics during behavioral changes, we compared the average values of the three basic information dynamics per behavioral epoch or behavioral state (Figure $2 \mathrm{~A}-\mathrm{C}$ ). Kruskal-Wallis analysis of variance found a small, but significant difference between all four behavioral conditions for AIS $\left(H=67, p=4.27 \times 10^{-15}\right)$. The effect size change in AIS values, however, was very small: the highest value was $0.014 \pm 0.024$ (in the fixation condition), while the smallest was $0.012 \pm 0.021$ (Cohen's $\mathrm{D}=0.1$ ). This suggests that during the movement condition, the degree to which information is being "stored" in individual neurons is decreased, possibly in favor of information flowing from sources to targets. There was a stronger, significant difference in mTE between behavioral states $\left(H=1555.649, p<10^{-20}\right)$. Here the lowest average mTE was observed in the fixation period $(0.005 \pm 0.007)$ and the highest was observed in the movement period $(0.013 \pm 0.02$, Cohen's $\mathrm{D}=0.46)$. The strongest significant difference was found with synergy $\left(H=1520.213, p<10^{-20}\right)$, with the smallest synergy being seen in the memory condition $(0.001 \pm 0.002)$ and the highest, as expected, being seen in the movement state $(0.002 \pm 0.003$, Cohen's $\mathrm{D}=0.54)$. Time-resolving the behavioral states to the level of individual windows (Fig. 2, D-F) shows that, within behavioral states, the distributions of all three information dynamics were largely similar. The only exception was the memory state, where the mTE and the synergy started to increase before the onset of movement. This is however to be expected because movement-related processing in cortical motor areas precedes movement onset by at least the amount of neuronal distance to the arm and hand [31, 32].

\subsection{1 "Dynamical Morphospace" Embedding}

Each of the three information dynamics described above describes an independent "mode" of information processing. We used these measures to construct a "dynamical morphospace" [3, 30], which allows us to visualize global differences between states by using a non-linear manifold dimension reduction algorithm (UMAP [28]). Dynamically similar systems being geometrically "closer" in the embedded space. For every window, we embedded all of the neurons in a four-dimensional space paramaterized by: AIS, average mTE, average synergy, and average redundancy and then used UMAP to visualize this high-dimensional space (see Fig. 2G). We can see that the memory behaviour and the movement behaviour generally occupy distinct (but overlapping) regions of the configuration space. Thus, large difference in information dynamics are present between the different behavioral states of the whole network, despite only small observable differences between fixation, cue and memory in average AIS, mTE and synergy. 


\subsubsection{Redundancy / Synergy Bias}

The measure of information modification (synergy) also reveals an additional set of information processing "modes", formalized by the partial information decomposition (PID) framework [33, 34] which reveals two different ways information can flow through a system: a redundancy-dominated mode, where information is duplicated and sent through many channels simultaneously, or a synergy-dominated mode, where statespecific processing occurs by transforming inputs into synergistic outputs. These distinct modes are thought to play different roles in macro-scale brain function and behavior [35, 36], although the computational role they play at the level of neural networks remains unclear.

The PID framework shows that the mutual information between two sources $\left(S_{1}, S_{2}\right)$ and a $\operatorname{target} T$ can be broken down into additive, non-overlapping components: redundant information (information about the target that both sources disclose), unique information (information about the target only disclosed by one source), and synergistic information (information about the target only disclosed by the joint state). We can assess which of these modes dominates the overall joint mutual information $I\left(S_{1}, S_{2} ; T\right)$ by normalizing the value of each atom (i.e. the portion of the joint-MI that is redundant, unique, or synergistic) by the total mutual information. Note that this normalization is different from the one reported above (where the value of every information dynamic is normalized by the entropy of the receiver neuron). We found that, in all behavioural states, the information flow was synergy dominated (see Fig. 2H-I), and that for the first three behavioral states (fixation, cue, memory), the values of synergy and redundancy as well as their ratio remained relatively constant. The transition from memory to movement, however, was associated with a marked increase in the relative redundancy of information flow $(\Delta=-74.4 \pm-27.3 \%, \mathrm{U}=10084455.0$, $\mathrm{p}<10^{-10}$ ). No other transition (fixation $\rightarrow$ cue, or cue $\rightarrow$ memory) was significant, despite the very large number of samples in each distribution. This indicates that the transition from "cognitive" states to motor states is associated with a particular increase in the relative dominance of redundant information communicated within the network.

When assessing the proportion of joint mutual information attributable to individual partial information atoms (e.g. the redundant, unique, and synergistic components of the joint mutual information), KruskalWallis analysis of variance found a small, but significant difference between behavioral states for proportional synergy $(H=11.287 p=0.01)$, with the smallest values appearing in the fixation condition $(0.206 \pm 0.14)$ and the highest value appearing in the cue condition $(0.226 \pm 0.189$, Cohen's $D=0.226 \pm 0.189)$. For visualization see Figure 2I. This very small effect size, in contrast to the earlier-reported synergy result highlights how different information dynamics can tell different stories depending on context: previously, the synergy reported was given as a percentage of the entropy of the receiver neuron explained by synergistic information flow. Here it is instead the proportion of the joint mutual information from sources to targets - these subtle differences reveal how the same measures can provide different insights in different contexts. Unlike proportional synergy, there were large, significant differences between behavioral states with respect to the proportion of joint mutual information explained by redundancy $\left(H=634.614 p<10^{-10}\right)$, with the lowest proprotional redundancy appearing during the fixation condition $(0.131 \pm 0.147)$, and climbing to a maximum in the movement condition $(0.203 \pm 0.152$, Cohen's $\mathrm{D}=0.478)$. The majority of all the joint mutual information was provided uniquely by one of the two parents, which remained largely constant throughout the behavioral states. Taken together, these results show consistent differences between different behavioral states in terms of the kinds of information-processing dynamics being carried out by the neural networks. We can see that the onset of movement is associated with a significant increase in both the amount of information flowing through the system as well as the amount of "computational" information 
modification occurring. The information that is flowing through the system (as indicated by the transfer entropy) has an increased redundancy component, suggesting that the same information is being duplicated and sent through the system multiple times in parallel, possibly to ensure high-fidelity transmission to the muscles for successful execution of the task.

\subsubsection{Network Dynamics}

The three information dynamics provide a hierarchy of increasingly higher-order interactions between neurons, however the structure of the partial information decomposition (PID) limits us to synergistic relationships between three neurons. The dynamic morphospace analysis suggests that higher-order, global relationships between neurons exist. To assess this global collective structure in fine detail, we analyzed the global network topology [18, 19], examining how how the global structure of the effective connectivity network changed over time. By time-resolving the analysis of network topology for every window, we observed that the onset of the cue is associated with a transient reconfiguration of the network structure (see Fig. 3A-C). While the density of the network did not significantly change in response to cue onset, nonparametric cluster-based surrogate testing (See Sec. 5.7.3 [37, 17] revealed that, for both grip-types A (precision grip) and B (power grip), the windows during which the cue was on had a significantly higher normalized clustering coefficient $\left(p<10^{-20}\right.$ for all windows and all grip types) than would be expected by chance. Similarly, for both grip types, the cue-on windows had a significantly higher rich-club coefficient $(p=0.002$ for Grip A and $p=0.048$ for Grip B). We can also see a significant increase in the rich club coefficient for both grip types in the movement state ( $p<10^{-20}$ for all windows and all grip-types). The increase in the rich club coefficient during the movement condition was one of the strongest effects, strongly suggesting that different cognitive and behavioral states are associated with changes to the global information-processing architecture.

This shows that the transition from a passive cognitive state (fixation, memory) to an active cognitive state (perception of a visual cue or motor execution) is associated with marked changes to the global topology of the information processing network in a way that is not revealed when considering the information dynamics of individual neurons (see Fig. 2 D-F for comparison). For example, an increase in rich-club structure is seen in both the cue period and during movement execution, suggesting that the presence of rich-club structure is important in particular for the active execution of a task. Interestingly, this re-configuration of the network occurs during the cue-on epoch without a corresponding change in the density of the network, suggesting that it might be a true re-allocation of a fixed quantity of computational resources.

\subsubsection{AIS and mTE Similarity Clustering}

A crucial element of this study is that the macaques were randomly assigned one of two possible grasp conditions for every trial. This allows us to assess fine-grained differences in the information dynamics of networks of neurons for different perceptual, cognitive and behavioral conditions during the same states. To assess this we examined how the distribution of AIS values across neurons, and mTE values across network edges, varied between grip types. For both measures, we created cosine-similarity matrices for every pair of time windows for every behavioral state and both grip-types (see Fig. 3D-E). The windows were ordered in time (e.g. Fixation (Grip A) $\rightarrow$ Cue (Grip A) $\rightarrow$... Move (Grip A) $\rightarrow$ Fixation (Grip B) $\rightarrow$... etc) (see the ordering on the axes of Fig. 4D-E). By examining the off-diagonals (where the two grip-types are not the same), we can observe that the distribution of AIS and mTE values across the recordings were more similar during the fixation epoch, and then the systems diverged following the completion of the cue (the red squares in the referenced figure). We can see that after the onset of the cue, the similarity of the networks decreased, 


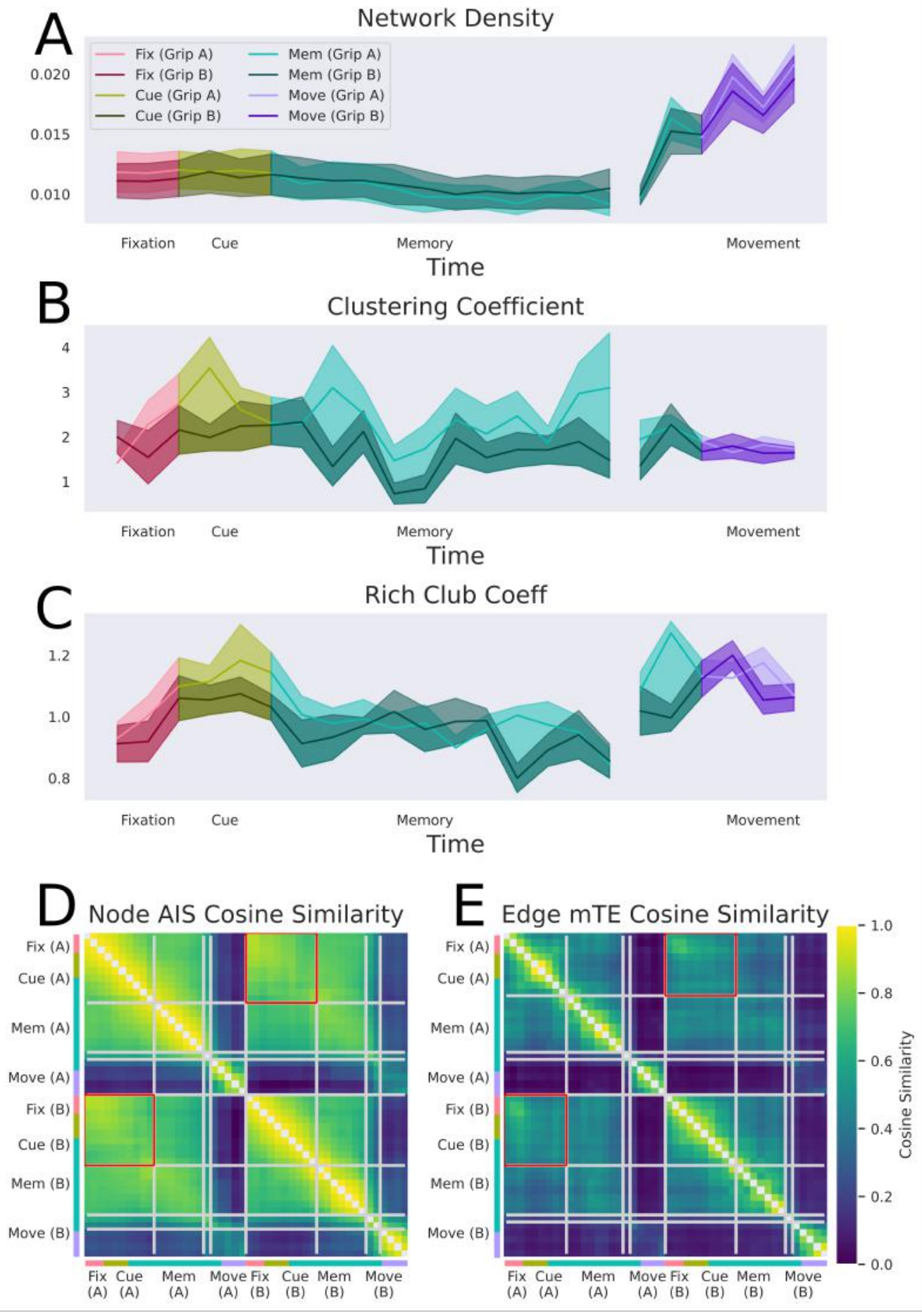

Figure 3: Differences in effective connectivity networks between task states and conditions. A-C Window-level time-resolved time-series for the three measures of network topologies: network density (A), normalized global clustering coefficient (B), and the normalized rich club coefficient (C). The network density increased dramatically in the movement epoch but remained constant during all "cognitive" states. In contrast, the networks showed a transient reconfiguration during the cue epoch: increasing the hierarchical rich-club structure and becoming more clustered. D-E Pairwise cosine similarity matrices for the distribution of AIS and mTE values for all windows. Grey lines indicate the boundaries of similar clusters as determined with a clustering algorithm. When data is arranged by behavioral sequence and grip type, we can see that the patterns of activity prior to perception of a visual cue are highly similar, but this similarity vanishes after the end of the cue epoch. Also note that the inferred clusters map onto boundaries between different behaviors. 
and that there was a strong condition-dependent difference between the first three conditions (fixation, cue, memory) and the movement condition. This shows that different states can be distinguished at different levels of structural similarity: large difference e.g. the transition between memory and movement) and small differences (e.g. the similarities between pre-cue fixation epochs.)

To statistically verify this, we used a multi-resolution consensus clustering algorithm [38] (see Sec. 5.8)), which determined the existence of a community of these early-epoch, off-diagonal nodes. This is to be expected, as the fixation phase is the only state that is independent of the cued-behavioral condition and consequently shows patterns common to both. It can be seen that the MRCC algorithm found clusters that correspond to distinct behavioral states (cluster boundaries are delineated with grey lines in Figure 3D-E).

For both information dynamics measures, it is clear that, when the grip types differ, the similarity of the effective network structure is highest during the fixation/waiting behaviour: in both cases, the monkey does not know what the coming cue indicates, and so revert to a kind of baseline. Once the cue turns off, the similarity of the effective network structure drops, indicating that the holding of different commands in working memory involves the formation of distinct "information processing architectures" that can be discriminated.

\subsection{Relating Structure \& Functional Information Dynamics}

Previous studies performed on organotypic brain slices showed that synergistic information dynamics were highest between neurons with a high degree $[13,14,15]$. Yet, the meaning of synergistic processes in neural networks remains unclear. Therefore it is important to scrutinise this relationship in the intact brain during behaviorally relevant processing. To assess this, we correlated each of the information dynamics for every neuron against local network measures (local clustering coefficient and in-strength, as a proxy for local rich club). We found no significant relationships between either in-strength or local clustering coefficient and node-level AIS. However, we found a strong, significant correlation between the in-strength of a neuron and the average mTE flowing into it $\left(\mathrm{r}=0.86, \mathrm{p}<10^{-10}\right)$. While this is unsurprising, it is not entirely trivial: a low in-degree neuron and a high in-degree node might have the same in-strength, but different average incoming transfer entropies. Similarly, we found a strong, significant correlation between the in-strength of a neuron and its local clustering coefficient $\left(\mathrm{r}=0.44, \mathrm{p}<10^{-10}\right)$, which indicates that the information processing occurring in a single neuron is informed by the local connectivity pattern of that neuron's neighbors. Finally, we found strong, significant correlations between synergy and both in-strengths $\left(\mathrm{r}=0.657, \mathrm{p}<10^{-10}\right)$ and local clustering coefficient $\left(\mathrm{r}=0.653, \mathrm{p}<10^{-10}\right)$. As with the transfer entropy results, these results show that a neuron's location in the effective connectivity network relative to other neurons, informs the kinds of computations it performs.

\subsection{Anatomical Localization}

Every neuron recorded from can be identified as belong to one of three distinct brain regions: M1, AIP, and F5 (see Sec. 5.3). By breaking down analyses by brain regions, we can determine how changes in dynamical regimes vary by brain region. We can observe that different regions have different "baselines", as well as responding to changes in condition to greater or lesser extents (for a visualization of all the results, see Fig. 5). during fixation, the M1 region begins with a significantly lower level of synergy $(0.00013 \pm 0.003)$ then either AIP $\left(0.0011 \pm 0.003\right.$, Mann-Whitney $\left.U=13151.0, \mathrm{p}<10^{-10}\right)$ or F5 $\left(0.001 \pm 0.001, \mathrm{U}=9871966.0, \mathrm{p}<10^{-10}\right)$.

In contrast, during the movement condition, the M1 region had the highest synergy $(0.003 \pm 0.004)$ compared 

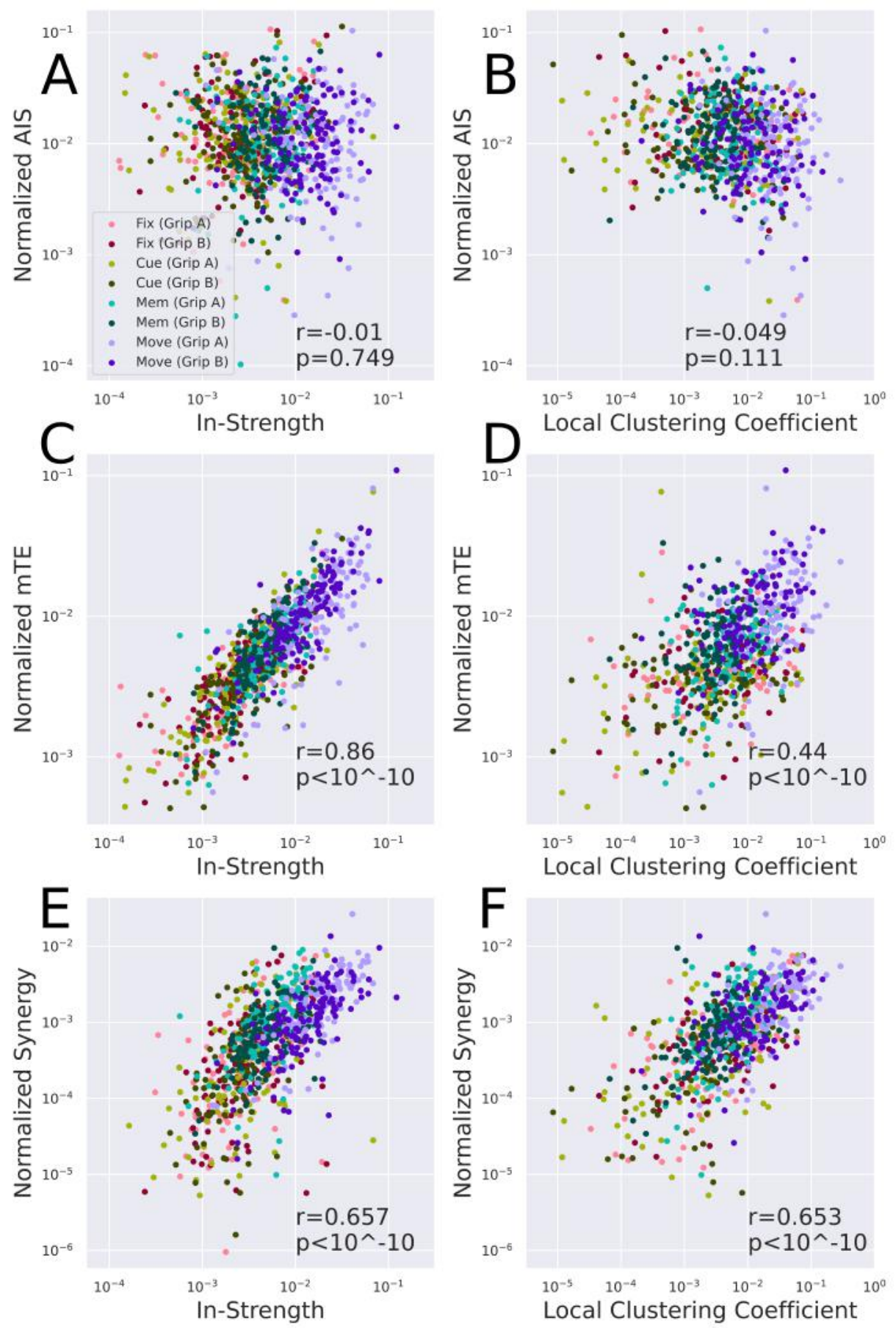

Figure 4: Information dynamics \& local network structure. Plots showing how node-level information dynamics are related to node-level network measures, correlations measured with the non-parametric Spearmanm correlation coefficient. The color-schema is the same as in previous plots. A-B We found no significant relationship between active information storage and the in-strength of a node or the local clustering coefficient. $\mathbf{C}$ There is a highly significant correlation between the average transfer entropy of a node and its in-strength $\left(r=0.869, p<10^{-10}\right.$, which is unsurprising as both measures quantify the total information flow into a neuron, albeit in slightly different ways. D There is a significant correlation between average transfer entropy and local clustering coefficient $\left(r=0.44, p<10^{-10}\right)$, which shows that the local community structure a neuron is embedded in implies where information flow is maximal. E The triadic synergy is positively correlated with the in-strength of target neuron $\left(r=0.657, p<10^{-10}\right)$, replicating the findings of [13]. F The triadic synergy is positively correlated with the local clustering coefficient $\left(r=0.653, p<10^{-10}\right)$, suggesting that extended local neighborhood structure plays a role in computation. 
to both AIP $\left(0.001 \pm 0.002\right.$, Mann-Whitney $\left.\mathrm{U}=748480.0, \mathrm{p}<10^{-10}\right)$ and F5 $(0.002 \pm 0.004$, Mann-Whitney $\mathrm{U}=2014222.0, \mathrm{p}=0.0004$ ). A similar result can be seen when examining the redundancy/synergy bias for each region. For visualization, see Figure 5A-D. This shows strong evidence that, not only do different brain regions instantiate different computational "regimes", but that they change in distinct ways in response to the demands of performing particular tasks.

In addition to the information dynamics measures, anatomical localization allows us to assess how particular regions contribute to the overall global network structure. We found that, during the fixation, cue, and memory conditions, the rich club was comprised of roughly equal numbers of neurons from each brain region, although as the memory condition progressed, the number of rich-club neurons in M1 started to fall. By the onset of movement, however, the rich club was largely dominated by M1 neurons, followed by F5 neurons, and AIP neurons maintained approximately the same number of nodes participating as the other three conditions. For visualization see Figure 5E. This suggests that the network reconfiguration that occurs during movement is driven largely by an increase in integration in M1 and F5 regions, neglecting AIP. This result is in agreement with the stronger involvement of the premotor cortex (of which F5 is a part) and M1 during movement control then parietal areas (of which AIP is a part) [39, 40? ]. Moreover, this result offers a possible explanation for the shift in dominance toward these areas.

\section{Discussion}

In this work, we have explored how the "computational" properties of biological neural networks recorded from the brain of awake, behaving, macaques change when they are engaged in different cognitive and behavioral states and conditions (inactive rest, perception of a visual cue, preparation and memorizing of different grips, execution of different grips). We decomposed "computation" into three, distinct "modes" of information processing: the active information storage (how the past activity of a single neuron constrains its future), transfer entropy (how the past of a set of source neurons constrains a single target neuron's future), and the information modification/synergy (information from the joint-state of multiple sources about a target's future that is irreducible to any simpler combination of sources). These modes form a semi-orthogonal set of "axes of complexity" and can vary as behaviour or cognition changes. We found that distinct behavioural states are associated with distinct patterns of information dynamics, and that these patterns (such as the flow of multivariate transfer entropy across the system) can reveal global changes in the system (such as the altered rich-club structure) in consistent ways.

This work both replicates and extends, previous work on information dynamics in biological neural networks. Prior work has demonstrated the existence of synergistic processing in dissociated cultures [9, 14], and that synergistic dynamics can be sensitive to local network structure [27] and vary over time [10]. Similar analyses have been done for AIS [4] and TE [5, 6], although until it has been a mystery how these different modes of computation relate to cognition and behavior. We found that the onset of the movement behaviour was associated with a mild decrease in active information storage, and an increase in both information flow and information modification, although different brain regions showed different degrees of responsiveness. For example, the decrease in AIS was most pronounced in the F5 region (which also had the lowest baseline AIS), while the increase in synergy was most pronounced in the M1 region (which interestingly, began with the lowest baseline synergy).

A key finding in this report is that not only do individual information modes change for different cognitive and behavioural states as well as areas, but the atomic components of information flow can vary in dominance: 

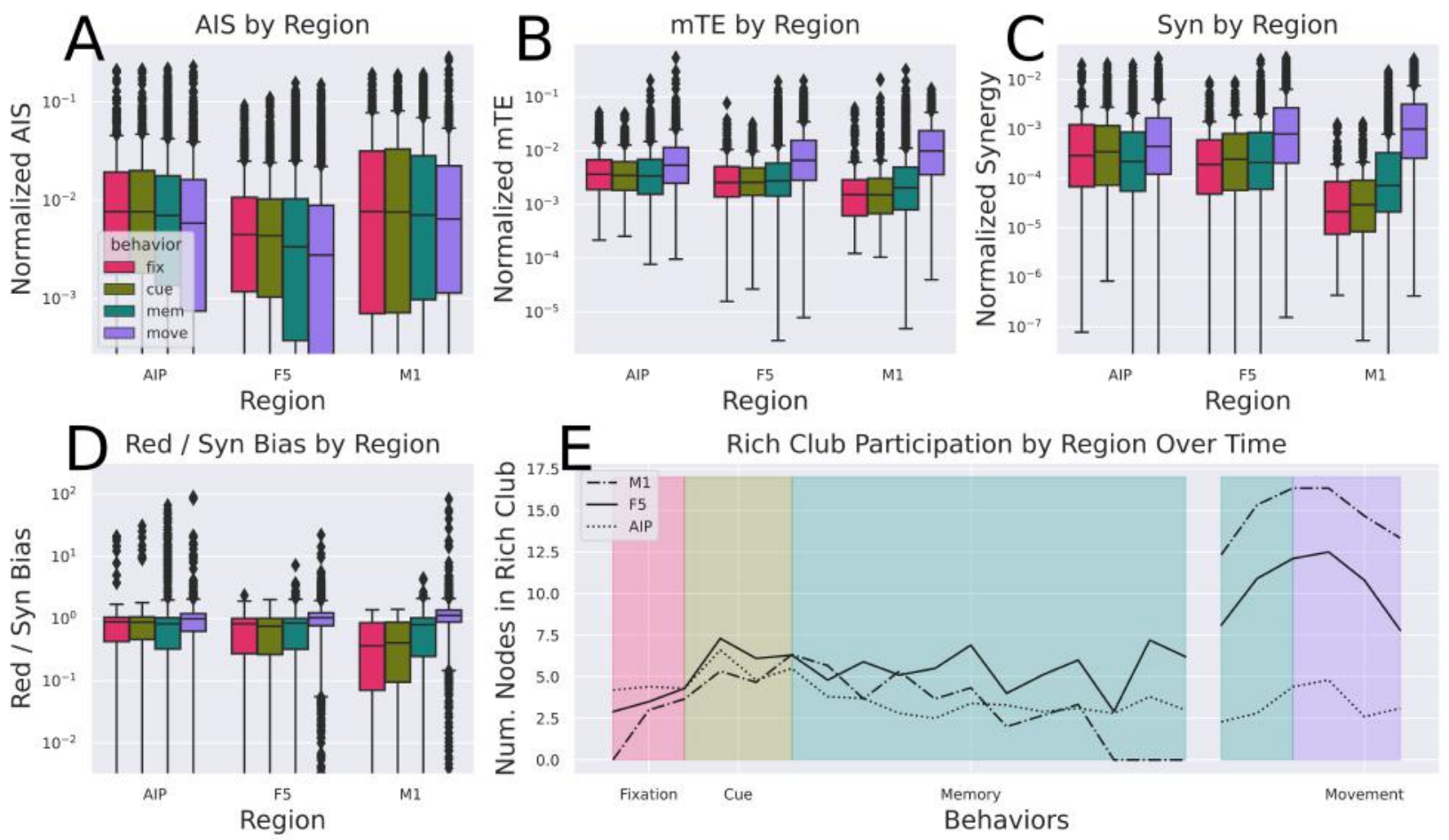

Figure 5: Brain-region specific information profiles. A-C: The breakdown of active information storage (A), multivariate transfer entropy $(\mathbf{B})$, and synergy $(\mathbf{C})$ by the four behavioral states (colors) and the three recorded regions (AIP, M1, F5). We can see that information dynamics in different brain regions have different levels of responsiveness to differed states. D The ratio of redundancy/synergy dominance for all behavioral epochs and all brain regions. While less pronounced then the standard information dynamics, distinct differences between brain regions are visible. $\mathbf{E}$ The number of nodes from each of the three brain regions that participate in the rich club over time. We can see that, on average, each region initially contributes equally, however during the onset of movement, the M1 and F5 regions become significantly more engaged, while the AIP does not. 
this is indicated by the significant increase in redundancy relative to synergy during the onset of movement. While the system generally remains synergy-dominated, the relative increase in redundancy (particularly in M1) suggests an intuitive model: M1 serves as a motor "output region", connecting to the rest of body and its general "role" is to coordinate successful completion of motor outputs as hypothesised in previous studies [41, 40, 42, 43, 44] In a situation requiring a high degree of error-free signal transmission, an increase in redundant information may improve the odds of successfully completing the required behaviour (as redundancy builds resiliency into the channel). An appealing next step is to attempt to model how the system manages to execute this shift. Recent work has shown that the information processing structure of a biological neural network is sensitive to the particular dynamical regime the system is in, with criticality maximizing information-theoretic complexity [9], and that this relationship tracks deviations from normal cognitive processing [45]. Similarly, changes in synergy and redundancy track changes towards and away from the critical regime [46], which suggests that one mechanism by which the brain may regulate the relative synergyor redundancy-dominance of activity is by altering its "position" relative to a critical phase transition.

Another intriguing finding is that the act of holding different symbols in memory corresponds to distinct patterns of ongoing information-dynamic activity that are themselves distinct from the fixation/waiting condition. While the issue of "representation" in the brain is a deeply contentious one [47, 48] and beyond the scope of this work, these results strongly indicate that different visual cues (indicating different grip types) are associated with distinct patterns of information processing. The exact nature of these patterns, and what (if any) causal relationship they have with the successful completion of the required behaviour remains unclear and is a promising area for further study. One possible direction forward may be leveraging the framework of representational similarity analysis (RSA) [49, 50], finds common "representational spaces" of activity in the cortex. RSA is typically used in the context of fMRI data, where high spatial resolution is available, however information-theoretic approaches to binary spiking data may provide considerable insight into how information is "represented" at the meso-scale level of biological neural networks.

This study has some limitations that should be discussed, particularly in the context of replication and generalization. One of the most significant limitation is that, while multivariate transfer entropy gives a much more "complete" picture of the information transfer dynamics (and appears to better estimate the generative network [24]), it suffers from a terrible curse of dimensionality and will not scale gracefully as the number of neurons being recorded from gets large and/or the number of bins of history increases. Consequently, for researchers interested in the role of redundancies and synergies in timeseries dynamics, heuristics such as the O-information may be useful [51, 46, 52]. Another possibility might be initially inferring a bivariate transfer entropy network, and then restricting the prospective source set of each node to the sources inferred in the bTE network. While this would miss any purely synergistic relationships, it seems reasonable to assume that such relationships are rare and that effective connections are composed of a full complement of redundant, unique, and synergistic modes, so all statistically significant edges would be "seen" by the bTE algorithm (although they could be winnowed out by serial conditioning). The bTE-inferred parent set might then be considered a maximally optimistic set, which can be fine-tuned using the mTE network inference algorithm with the restricted possible source set.

The partial information decomposition (PID) framework suffers from a similar curse of dimensionality as the the mTE algorithm: the number of unique partial information atoms grows with the Dedekind number of sources, so while a triad of two sources and one target only has 4 atoms, a set of eight sources synapsing onto one target has $\approx 5.61 \times 10^{22}$ distinct partial information atoms. Since neurons can participate in hundreds or thousands of synaptic relationships, being restricted to sets of 1-5 interacting neurons is a severe limitation. 
Consequently, there is an urgent need for analytic techniques to identify synergistic relationships that do not require brute-forcing the entire partial information lattice. One possible way forward is a direct estimation of synergistic integration via stochastic intermediary variables [53]. For estimating unique information, an intriguing relationship between unique information and public-key cryptography has been similarly proposed $[54,55]$.

There is a long-standing question about how best to address the issue of variable firing rates in neural recordings. One could argue that firing rate represents a confound that must be "conditioned out": for example, a higher-firing rate, but random, pair of neurons will likely have greater apparent transfer entropy relative to a lower firing-rate pair, simply because there is more entropy in the system overall, even if no relationship actually exists [6]. Alternatively one could argue that the firing rate is the channel: changing the level of neuronal activity appears to be at least one way that neurons communicate information (known as "rate coding", evidence for this mode dates back to the early days of neuroscience [56]). Alternative coding schema (such as temporal coding [57]) which rely on relative timings between neurons still can make use of changes in firing rate since higher entropy processes can support a wider range of firing rate patterns, including relatively low-probability patterns. The question of how best to, or whether to control for firing rate at all becomes ambiguous, especially since the exact nature of the neural code remains mysterious and likely combines many different dimensions including rates, spiking timing dependent codes, and more [58, 59]. This study demonstrates two fairly liberal normalizations: for the individual information dynamics results (AIS, mTE, synergy), we normalized the raw information values by the entropy of the receiver neuron to approximate the proportion of uncertainty explained by each mode. For sparse processes, this serves as a simple firing-rate correction, since low-firing rate neurons will have low entropy and vice versa for relatively high-firing rate neurons. Crucially, this simple correction is easily interpretable, it only requires informationtheoretic measures (keeping the analysis conceptually simple), and computationally practical (not requiring huge numbers of null models as in the AIS and mTE inference). The second normalization is done in the redundancy/synergy bias calculation, where each partial information atom is normalized by the total mutual information $I\left(S_{1}, S_{2} ; T\right)$. This provides a different interpretation of the information: rather than entropy explained, it is the proportion of the total information flow that is shared or synergistic, and not specific to any single source or target neuron. These different analytic choices throw different statistical relationships into the spotlight and highlight how it can be limiting to consider only one "true" correction and instead keep the pipeline flexible. Our hope is that the work presented here casts new insights into how the brain processes information, both about its environment and its own internal processes (such as correctly outputting a motor behavior). Beyond understanding the mammalian nervous system, these results may be of interest to work related to the development of artificial spiking neural networks. Previous discussion of information dynamics has argued that, in addition to providing a descriptive framework of recording activity, engineering particular information dynamics in artificial systems can help achieve effective biologically-inspired computing [60, 61], such as low-energy spiking artificial neural networks [62, 63, 64, 65].

In total, information dynamics represents an appealing framework with which to explore the brain writ large, and can be easily adapted to assess computational processes at multiple scales. Given the extensive public data available to neuroscientists, we are optimistic that information dynamics can provide new insights into the relationship between brain, behavior, computation, and dynamics. In particular, information dynamics marries two well-established frameworks in modern neuroscience: computational approaches, and dynamical systems approaches [66, 67, 32, 68, 43, 69]. Sometimes called "computational mechanics" [70], information dynamics analysis can provide a bridge between approaches to provide insights that may not be 
obvious from either one.

\section{Conclusion}

In this study, we show that the computational role of cortical neurons in awake, behaving macaques varies depending on cognitive and behavioural states and condition. These changes can be observed at the level of the temporal dependence of an individual neuron (active information storage), bivariate source to target interactions between neurons (transfer entropy), and higher-order interactions (synergy, global network topology). Different cognitive and behavioural states (including the "cognitive processing" vs "movement execution" distinction and the "active" vs. "passive" distinction), are associated with distinct, multidimensional information-dynamic profiles, which can be visualized as a "dynamical morphospace." These results extend prior work that has been done assessing information dynamics in non-cognitive model systems such as organotypic cultures, and reveals how changes in processing regimes can inform on how the brain implements complex cognition and behaviors. This work has implications both for our understanding of how "computation" emerges from the decentralized interaction of neurons, as well as how we may be able to engineer effective, energy efficient, neuromorphic computing architectures.

\section{Author Contributions}

TFV, OS, and BD collectively conceptualized the project. HS and BD prepared experiments. BD collected and preprocessed the data. TFV, OS, and BD performed analysis of the data. TFV wrote the initial draft of the manuscript. OS, HS and BD provided feedback and editing of the manuscript. All authors approved final submission.

\section{Competing Interests}

The authors declare no competing interests.

\section{Acknowledgements}

TFV is supported by NSF-NRT grant 1735095, Interdisciplinary Training in Complex Networks and Systems. TFV and OS are supported by NIH R01 MH121978. and BD and HS are supported by the German Minstery of Education and Research (FKZ 01GQ1903). We would like to thank Natalie Bobb, Ricarda Lbik Mathias Dörge and Dr. Stefan Schaffelhofer for their assistance with animal handling, setup maintenance and data recording. Moreover. We would like to thank Dr. Joe Lizier for advice throughout the process, particularly in regards to the use of the IDTxl package, as well as Dr. Patricia Wollstadt for her help as the maintainer of the IDTxl repository, and all of the other developers who contributed. Furthermore we would like to thank Dr. Samantha Sherrill for her insight and experience using PID in the context of single neuron recordings, as well as Drs. John Beggs and Ehren Newman and their respective labs for insightful feedback. 


\section{References}

[1] Peter Sterling and Simon Laughlin. Principles of Neural Design. MIT Press, June 2015. Google-BooksID: VVqrCQAAQBAJ.

[2] M. Mitchell. Non-standard computation: molecular computation, cellular automata, evolutionary algorithms, quantum computers. Wiley-VCH, Weinheim, New York, 1998. Open Library ID: OL456861M.

[3] Joseph T. Lizier, Mikhail Prokopenko, and Albert Y. Zomaya. A Framework for the Local Information Dynamics of Distributed Computation in Complex Systems. In Mikhail Prokopenko, editor, Guided Self-Organization: Inception, Emergence, Complexity and Computation, pages 115-158. Springer, Berlin, Heidelberg, 2014.

[4] Michael Wibral, Joseph Lizier, Sebastian Vögler, Viola Priesemann, and Ralf Galuske. Local active information storage as a tool to understand distributed neural information processing. Frontiers in Neuroinformatics, 8, 2014. Publisher: Frontiers.

[5] Michael Wibral, Raul Vicente, and Michael Lindner. Transfer Entropy in Neuroscience. In Michael Wibral, Raul Vicente, and Joseph T. Lizier, editors, Directed Information Measures in Neuroscience, Understanding Complex Systems, pages 3-36. Springer, Berlin, Heidelberg, 2014.

[6] Terry Bossomaier, Lionel Barnett, Michael Harré, and Joseph T. Lizier. An Introduction to Transfer Entropy: Information Flow in Complex Systems. Springer, November 2016. Google-Books-ID: p8eADQAAQBAJ.

[7] Benjamin Flecker, Wesley Alford, John M. Beggs, Paul L. Williams, and Randall D. Beer. Partial information decomposition as a spatiotemporal filter. Chaos: An Interdisciplinary Journal of Nonlinear Science, 21(3):037104, September 2011. Publisher: American Institute of Physics.

[8] J. T. Lizier and M. Prokopenko. Differentiating information transfer and causal effect. The European Physical Journal B, 73(4):605-615, February 2010.

[9] Nicholas M. Timme, Najja J. Marshall, Nicholas Bennett, Monica Ripp, Edward Lautzenhiser, and John M. Beggs. Criticality Maximizes Complexity in Neural Tissue. Frontiers in Physiology, 7, 2016.

[10] Michael Wibral, Conor Finn, Patricia Wollstadt, Joseph T. Lizier, and Viola Priesemann. Quantifying Information Modification in Developing Neural Networks via Partial Information Decomposition. Entropy, 19(9):494, September 2017. Number: 9 Publisher: Multidisciplinary Digital Publishing Institute.

[11] Thomas M. Cover and Joy A. Thomas. Elements of Information Theory. John Wiley \& Sons, November 2012. Google-Books-ID: VWq5GG6ycxMC.

[12] David P. Shorten, Viola Priesemann, Michael Wibral, and Joseph T. Lizier. Early lock-in of structured and specialised information flows during neural development. bioRxiv, page 2021.06.29.450432, June 2021. Publisher: Cold Spring Harbor Laboratory Section: New Results. 
[13] Nicholas M. Timme, Shinya Ito, Maxym Myroshnychenko, Sunny Nigam, Masanori Shimono, FangChin Yeh, Pawel Hottowy, Alan M. Litke, and John M. Beggs. High-Degree Neurons Feed Cortical Computations. PLOS Computational Biology, 12(5):e1004858, May 2016. Number: 5 Publisher: Public Library of Science.

[14] Samantha P. Faber, Nicholas M. Timme, John M. Beggs, and Ehren L. Newman. Computation is concentrated in rich clubs of local cortical networks. Network Neuroscience, pages 1-21, September 2018.

[15] Samantha P. Sherrill, Nicholas M. Timme, John M. Beggs, and Ehren L. Newman. Correlated activity favors synergistic processing in local cortical networks in vitro at synaptically relevant timescales. Network Neuroscience (Cambridge, Mass.), 4(3):678-697, 2020.

[16] Thomas Schreiber. Measuring Information Transfer. Physical Review Letters, 85(2):461-464, July 2000. Number: 2.

[17] Benjamin Dann, Jonathan A Michaels, Stefan Schaffelhofer, and Hansjörg Scherberger. Uniting functional network topology and oscillations in the fronto-parietal single unit network of behaving primates. eLife, 5:e15719, August 2016. Publisher: eLife Sciences Publications, Ltd.

[18] Olaf Sporns. Networks of the Brain. MIT Press, October 2010.

[19] Alex Fornito, Andrew Zalesky, and Edward Bullmore. Fundamentals of Brain Network Analysis. Academic Press, March 2016.

[20] Nicholas M. Timme and Christopher Lapish. A Tutorial for Information Theory in Neuroscience. eNeuro, 5(3):ENEURO.0052-18.2018, May 2018. Number: 3.

[21] Fei He and Yuan Yang. Nonlinear System Identification of Neural Systems from Neurophysiological Signals. Neuroscience, 458:213-228, March 2021.

[22] David Beniaguev, Idan Segev, and Michael London. Single cortical neurons as deep artificial neural networks. Neuron, August 2021.

[23] Leonardo Novelli, Patricia Wollstadt, Pedro Mediano, Michael Wibral, and Joseph T. Lizier. Largescale directed network inference with multivariate transfer entropy and hierarchical statistical testing. Network Neuroscience, 3(3):827-847, July 2019. Number: 3.

[24] Leonardo Novelli and Joseph T. Lizier. Inferring network properties from time series using transfer entropy and mutual information: Validation of multivariate versus bivariate approaches. Network Neuroscience, 5(2):373-404, May 2021.

[25] Patricia Wollstadt, Joseph T. Lizier, Raul Vicente, Conor Finn, Mario Martinez-Zarzuela, Pedro Mediano, Leonardo Novelli, and Michael Wibral. IDTxl: The Information Dynamics Toolkit xl: a Python package for the efficient analysis of multivariate information dynamics in networks. Journal of Open Source Software, 4(34):1081, February 2019. Number: 34.

[26] Joseph T. Lizier, Benjamin Flecker, and Paul L. Williams. Towards a Synergy-based Approach to Measuring Information Modification. arXiv:1303.3440 [nlin, physics:physics], March 2013. arXiv: 1303.3440. 
[27] Samantha P. Sherrill, Nicholas M. Timme, John M. Beggs, and Ehren L. Newman. Partial information decomposition reveals that synergistic neural integration is greater downstream of recurrent information flow in organotypic cortical cultures. PLOS Computational Biology, 17(7):e1009196, July 2021. Publisher: Public Library of Science.

[28] Leland McInnes, John Healy, and James Melville. UMAP: Uniform Manifold Approximation and Projection for Dimension Reduction. arXiv:1802.03426 [cs, stat], December 2018. arXiv: 1802.03426.

[29] Andrea Avena-Koenigsberger, Joaquín Goñi, Ricard Solé, and Olaf Sporns. Network morphospace. Journal of The Royal Society Interface, 12(103):20140881, February 2015. Number: 103 Publisher: Royal Society.

[30] Thomas F. Varley, Vanessa Denny, Olaf Sporns, and Alice Patania. Topological analysis of differential effects of ketamine and propofol anaesthesia on brain dynamics. Royal Society Open Science, 8(6):201971, June 2021. Publisher: Royal Society.

[31] Mark M. Churchland, John P. Cunningham, Matthew T. Kaufman, Stephen I. Ryu, and Krishna V. Shenoy. Cortical Preparatory Activity: Representation of Movement or First Cog in a Dynamical Machine? Neuron, 68(3):387-400, November 2010. Publisher: Elsevier.

[32] Jonathan A. Michaels, Benjamin Dann, and Hansjörg Scherberger. Neural Population Dynamics during Reaching Are Better Explained by a Dynamical System than Representational Tuning. PLOS Computational Biology, 12(11):e1005175, November 2016. Publisher: Public Library of Science.

[33] Paul L. Williams and Randall D. Beer. Nonnegative Decomposition of Multivariate Information. arXiv:1004.2515 [math-ph, physics:physics, q-bio], April 2010. arXiv: 1004.2515.

[34] Paul L. Williams and Randall D. Beer. Generalized Measures of Information Transfer. arXiv:1102.1507 [physics], February 2011. arXiv: 1102.1507.

[35] Andrea I. Luppi, Pedro A. M. Mediano, Fernando E. Rosas, Negin Holland, Tim D. Fryer, John T. O'Brien, James B. Rowe, David K. Menon, Daniel Bor, and Emmanuel A. Stamatakis. A synergistic core for human brain evolution and cognition. bioRxiv, page 2020.09.22.308981, September 2020. Publisher: Cold Spring Harbor Laboratory Section: New Results.

[36] Andrea I. Luppi, Pedro A. M. Mediano, Fernando E. Rosas, Judith Allanson, John D. Pickard, Robin L. Carhart-Harris, Guy B. Williams, Michael M. Craig, Paola Finoia, Adrian M. Owen, Lorina Naci, David K. Menon, Daniel Bor, and Emmanuel A. Stamatakis. A Synergistic Workspace for Human Consciousness Revealed by Integrated Information Decomposition. bioRxiv, page 2020.11.25.398081, November 2020. Publisher: Cold Spring Harbor Laboratory Section: New Results.

[37] Eric Maris and Robert Oostenveld. Nonparametric statistical testing of EEG- and MEG-data. Journal of Neuroscience Methods, 164(1):177-190, August 2007.

[38] Lucas G. S. Jeub, Olaf Sporns, and Santo Fortunato. Multiresolution Consensus Clustering in Networks. Scientific Reports, 8(1):3259, February 2018.

[39] Veera Katharina Menz, Stefan Schaffelhofer, and Hansjörg Scherberger. Representation of continuous hand and arm movements in macaque areas M1, F5, and AIP: a comparative decoding study. Journal of Neural Engineering, 12(5):056016, September 2015. Publisher: IOP Publishing. 
[40] Stefan Schaffelhofer and Hansjörg Scherberger. Object vision to hand action in macaque parietal, premotor, and motor cortices. eLife, 5:e15278, July 2016. Publisher: eLife Sciences Publications, Ltd.

[41] Mark M. Churchland, John P. Cunningham, Matthew T. Kaufman, Justin D. Foster, Paul Nuyujukian, Stephen I. Ryu, and Krishna V. Shenoy. Neural population dynamics during reaching. Nature, 487(7405):51-56, July 2012. Bandiera_abtest: a Cg_type: Nature Research Journals Number: 7405 Primary_atype: Research Publisher: Nature Publishing Group Subject_term: Motor cortex;Neuronal physiology;Population dynamics Subject_term_id: motor-cortex;neuronal-physiology;population-dynamics.

[42] Abigail A. Russo, Ramin Khajeh, Sean R. Bittner, Sean M. Perkins, John P. Cunningham, L. F. Abbott, and Mark M. Churchland. Neural Trajectories in the Supplementary Motor Area and Motor Cortex Exhibit Distinct Geometries, Compatible with Different Classes of Computation. Neuron, 107(4):745758.e6, August 2020.

[43] Jonathan A. Michaels, Stefan Schaffelhofer, Andres Agudelo-Toro, and Hansjörg Scherberger. A goaldriven modular neural network predicts parietofrontal neural dynamics during grasping. Proceedings of the National Academy of Sciences, 117(50):32124-32135, December 2020. Publisher: National Academy of Sciences Section: Biological Sciences.

[44] Kazutaka Takahashi, Sanggyun Kim, Todd P. Coleman, Kevin A. Brown, Aaron J. Suminski, Matthew D. Best, and Nicholas G. Hatsopoulos. Large-scale spatiotemporal spike patterning consistent with wave propagation in motor cortex. Nature Communications, 6(1):7169, May 2015. Bandiera_abtest: a Cc_license_type: cc_by Cg_type: Nature Research Journals Number: 1 Primary_atype: Research Publisher: Nature Publishing Group Subject_term: Motor cortex;Neural patterning Subject_term_id: motor-cortex;neural-patterning.

[45] Thomas Varley, Olaf Sporns, Aina Puce, and John Beggs. Differential effects of propofol and ketamine on critical brain dynamics. PLOS Computational Biology, 16(12):e1008418, December 2020. Number: 12 Publisher: Public Library of Science.

[46] Sebastiano Stramaglia, Tomas Scagliarini, Bryan C. Daniels, and Daniele Marinazzo. Quantifying Dynamical High-Order Interdependencies From the O-Information: An Application to Neural Spiking Dynamics. Frontiers in Physiology, 11, 2021. Publisher: Frontiers.

[47] Pim Haselager, Andre' de Groot, and Hans van Rappard. Representationalism vs. antirepresentationalism: A debate for the sake of appearance. Philosophical Psychology, August 2010. Publisher: Taylor \& Francis Group.

[48] Krishna V. Shenoy, Maneesh Sahani, and Mark M. Churchland. Cortical Control of Arm Movements: A Dynamical Systems Perspective. Annual Review of Neuroscience, 36(1):337-359, July 2013. Publisher: Annual Reviews.

[49] James. V. Haxby. Multivariate pattern analysis of fMRI: The early beginnings. Neuroimage, 62(2):852855, August 2012. Number: 2.

[50] J. Swaroop Guntupalli, Michael Hanke, Yaroslav O. Halchenko, Andrew C. Connolly, Peter J. Ramadge, and James V. Haxby. A Model of Representational Spaces in Human Cortex. Cerebral Cortex (New York, NY), 26(6):2919-2934, June 2016. Number: 6. 
[51] Fernando Rosas, Pedro A. M. Mediano, Michael Gastpar, and Henrik J. Jensen. Quantifying Highorder Interdependencies via Multivariate Extensions of the Mutual Information. Physical Review E, 100(3):032305, September 2019. Number: 3 arXiv: 1902.11239.

[52] Marilyn Gatica, Rodrigo Cofré, Pedro A.M. Mediano, Fernando E. Rosas, Patricio Orio, Ibai Diez, Stephan P. Swinnen, and Jesus M. Cortes. High-Order Interdependencies in the Aging Brain. Brain Connectivity, April 2021. Publisher: Mary Ann Liebert, Inc., publishers.

[53] Rick Quax, Omri Har-Shemesh, and Peter M. A. Sloot. Quantifying Synergistic Information Using Intermediate Stochastic Variables. Entropy, 19(2):85, February 2017. Number: 2 Publisher: Multidisciplinary Digital Publishing Institute.

[54] Ryan G. James, Jeffrey Emenheiser, and James P. Crutchfield. Unique information via dependency constraints. Journal of Physics A: Mathematical and Theoretical, 52(1):014002, December 2018. Number: 1 Publisher: IOP Publishing.

[55] Ryan G. James, Jeffrey Emenheiser, and James P. Crutchfield. Unique Information and Secret Key Agreement. Entropy, 21(1):12, January 2019. Number: 1 Publisher: Multidisciplinary Digital Publishing Institute.

[56] E. D. Adrian and Yngve Zotterman. The impulses produced by sensory nerve-endings. The Journal of Physiology, 61(2):151-171, April 1926.

[57] Wulfram Gerstner and Werner M Kistler. Spiking neuron models: single neurons, populations, plasticity. Cambridge University Press, Cambridge, U.K.; New York, 2002. OCLC: 57417395.

[58] Anil K. Seth. Neural Coding: Rate and Time Codes Work Together. Current Biology, 25(3):R110-R113, February 2015.

[59] Yanfang Zuo, Houman Safaai, Giuseppe Notaro, Alberto Mazzoni, Stefano Panzeri, and Mathew E. Diamond. Complementary Contributions of Spike Timing and Spike Rate to Perceptual Decisions in Rat S1 and S2 Cortex. Current Biology, 25(3):357-363, February 2015.

[60] Michael Wibral, Joseph T. Lizier, and Viola Priesemann. Bits from Brains for Biologically Inspired Computing. Frontiers in Robotics and AI, 2, 2015. Publisher: Frontiers.

[61] Ruomin Zhu, Joel Hochstetter, Alon Loeffler, Adrian Diaz-Alvarez, Tomonobu Nakayama, Joseph T. Lizier, and Zdenka Kuncic. Information dynamics in neuromorphic nanowire networks. Scientific

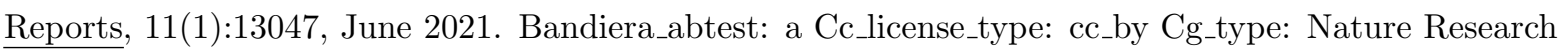
Journals Number: 1 Primary_atype: Research Publisher: Nature Publishing Group Subject_term: Complex networks;Dynamical systems;Electronic properties and materials;Information theory and computation;Nanowires;Network models;Nonlinear phenomena Subject_term_id: complex-networks;dynamicalsystems;electronic-properties-and-materials;information-theory-and-computation;nanowires;networkmodels;nonlinear-phenomena.

[62] Amirhossein Tavanaei, Masoud Ghodrati, Saeed Reza Kheradpisheh, Timothée Masquelier, and Anthony Maida. Deep learning in spiking neural networks. Neural Networks, 111:47-63, March 2019. 
[63] Kaushalya Kumarasinghe, Nikola Kasabov, and Denise Taylor. Deep learning and deep knowledge representation in Spiking Neural Networks for Brain-Computer Interfaces. Neural Networks, 121:169185, January 2020.

[64] Martino Sorbaro, Qian Liu, Massimo Bortone, and Sadique Sheik. Optimizing the Energy Consumption of Spiking Neural Networks for Neuromorphic Applications. Frontiers in Neuroscience, 0, 2020. Publisher: Frontiers.

[65] Priyadarshini Panda, Sai Aparna Aketi, and Kaushik Roy. Toward Scalable, Efficient, and Accurate Deep Spiking Neural Networks With Backward Residual Connections, Stochastic Softmax, and Hybridization. Frontiers in Neuroscience, 0, 2020. Publisher: Frontiers.

[66] Pedro A. M. Mediano, Fernando E. Rosas, Juan Carlos Farah, Murray Shanahan, Daniel Bor, and Adam B. Barrett. Integrated information as a common signature of dynamical and informationprocessing complexity. arXiv:2106.10211 [nlin, q-bio], June 2021. arXiv: 2106.10211.

[67] David Sussillo, Mark M. Churchland, Matthew T. Kaufman, and Krishna V. Shenoy. A neural network that finds a naturalistic solution for the production of muscle activity. Nature Neuroscience, 18(7):10251033, July 2015. Bandiera_abtest: a Cg_type: Nature Research Journals Number: 7 Primary_atype: Research Publisher: Nature Publishing Group Subject_term: Dynamical systems;Motor cortex;Network models Subject_term_id: dynamical-systems;motor-cortex;network-models.

[68] Chethan Pandarinath, Daniel J. O'Shea, Jasmine Collins, Rafal Jozefowicz, Sergey D. Stavisky, Jonathan C. Kao, Eric M. Trautmann, Matthew T. Kaufman, Stephen I. Ryu, Leigh R. Hochberg, Jaimie M. Henderson, Krishna V. Shenoy, L. F. Abbott, and David Sussillo. Inferring single-trial neural population dynamics using sequential auto-encoders. Nature Methods, 15(10):805-815, October 2018. Bandiera_abtest: a Cg_type: Nature Research Journals Number: 10 Primary_atype: Research Publisher: Nature Publishing Group Subject_term: Computational neuroscience;Machine learning;Motor control Subject_term_id: computational-neuroscience;machine-learning;motor-control.

[69] Saurabh Vyas, Matthew D. Golub, David Sussillo, and Krishna V. Shenoy. Computation Through Neural Population Dynamics. Annual Review of Neuroscience, 43(1):249-275, 2020 . _eprint: https://doi.org/10.1146/annurev-neuro-092619-094115.

[70] Cosma Rohilla Shalizi and James P. Crutchfield. Computational Mechanics: Pattern and Prediction, Structure and Simplicity. Journal of Statistical Physics, 104(3):817-879, August 2001.

[71] Jonathan A. Michaels, Benjamin Dann, Rijk W. Intveld, and Hansjörg Scherberger. Predicting Reaction Time from the Neural State Space of the Premotor and Parietal Grasping Network. Journal of Neuroscience, 35(32):11415-11432, August 2015. Publisher: Society for Neuroscience Section: Articles.

[72] P. G Musial, S. N Baker, G. L Gerstein, E. A King, and J. G Keating. Signal-to-noise ratio improvement in multiple electrode recording. Journal of Neuroscience Methods, 115(1):29-43, March 2002.

[73] R. Quian Quiroga, Z. Nadasdy, and Y. Ben-Shaul. Unsupervised Spike Detection and Sorting with Wavelets and Superparamagnetic Clustering. Neural Computation, 16(8):1661-1687, August 2004.

[74] Alexander Kraskov, Numa Dancause, Marsha M. Quallo, Samantha Shepherd, and Roger N. Lemon. Corticospinal Neurons in Macaque Ventral Premotor Cortex with Mirror Properties: A Potential Mechanism for Action Suppression? Neuron, 64(6):922-930, December 2009. 
[75] S. N. Gozani and J. P. Miller. Optimal discrimination and classification of neuronal action potential waveforms from multiunit, multichannel recordings using software-based linear filters. IEEE Transactions on Biomedical Engineering, 41(4):358-372, April 1994. Conference Name: IEEE Transactions on Biomedical Engineering.

[76] Antonis D. Savva, Georgios D. Mitsis, and George K. Matsopoulos. Assessment of dynamic functional connectivity in resting-state fMRI using the sliding window technique. Brain and Behavior, 9(4), March 2019.

[77] Joseph T. Lizier. The Local Information Dynamics of Distributed Computation in Complex Systems. Springer Theses. Springer Berlin Heidelberg, Berlin, Heidelberg, 2013.

[78] Floris Takens. Detecting strange attractors in turbulence. In David Rand and Lai-Sang Young, editors, Dynamical Systems and Turbulence, Warwick 1980, volume 898, pages 366-381. Springer Berlin Heidelberg, Berlin, Heidelberg, 1981. Series Title: Lecture Notes in Mathematics.

[79] A. Mason, A. Nicoll, and K. Stratford. Synaptic transmission between individual pyramidal neurons of the rat visual cortex in vitro. The Journal of Neuroscience: The Official Journal of the Society for Neuroscience, 11(1):72-84, January 1991.

[80] Peter Barthó, Hajime Hirase, Lenaïc Monconduit, Michael Zugaro, Kenneth D. Harris, and György Buzsáki. Characterization of Neocortical Principal Cells and Interneurons by Network Interactions and Extracellular Features. Journal of Neurophysiology, 92(1):600-608, July 2004. Publisher: American Physiological Society.

[81] Christopher J. Quinn, Todd P. Coleman, Negar Kiyavash, and Nicholas G. Hatsopoulos. Estimating the directed information to infer causal relationships in ensemble neural spike train recordings. Journal of Computational Neuroscience, 30(1):17-44, February 2011.

[82] Shinya Ito, Fang-Chin Yeh, Emma Hiolski, Przemyslaw Rydygier, Deborah E. Gunning, Pawel Hottowy, Nicholas Timme, Alan M. Litke, and John M. Beggs. Large-Scale, High-Resolution Multielectrode-Array Recording Depicts Functional Network Differences of Cortical and Hippocampal Cultures. PLOS ONE, 9(8):e105324, August 2014. Number: 8.

[83] Sunny Nigam, Masanori Shimono, Shinya Ito, Fang-Chin Yeh, Nicholas Timme, Maxym Myroshnychenko, Christopher C. Lapish, Zachary Tosi, Pawel Hottowy, Wesley C. Smith, Sotiris C. Masmanidis, Alan M. Litke, Olaf Sporns, and John M. Beggs. Rich-Club Organization in Effective Connectivity among Cortical Neurons. Journal of Neuroscience, 36(3):670-684, January 2016. Publisher: Society for Neuroscience Section: Articles.

[84] Nicholas Timme, Shinya Ito, Maxym Myroshnychenko, Fang-Chin Yeh, Emma Hiolski, Pawel Hottowy, and John M. Beggs. Multiplex Networks of Cortical and Hippocampal Neurons Revealed at Different Timescales. PLOS ONE, 9(12):e115764, December 2014. Publisher: Public Library of Science.

[85] Masanori Shimono and John M. Beggs. Functional Clusters, Hubs, and Communities in the Cortical Microconnectome. Cerebral Cortex, 25(10):3743-3757, October 2015.

[86] Mauro Ursino, Giulia Ricci, and Elisa Magosso. Transfer Entropy as a Measure of Brain Connectivity: A Critical Analysis With the Help of Neural Mass Models. Frontiers in Computational Neuroscience, 14, 2020. Publisher: Frontiers. 
[87] Nicholas Timme, Wesley Alford, Benjamin Flecker, and John M. Beggs. Synergy, redundancy, and multivariate information measures: an experimentalist's perspective. Journal of Computational Neuroscience, 36(2):119-140, April 2014. Number: 2.

[88] A Hagberg, D Schult, and P Swart. Exploring Network Structure, Dynamics, and Function using NetworkX. 2008.

[89] V. Colizza, A. Flammini, M. A. Serrano, and A. Vespignani. Detecting rich-club ordering in complex networks. Nature Physics, 2(2):110-115, February 2006. Bandiera_abtest: a Cg_type: Nature Research Journals Number: 2 Primary_atype: Research Publisher: Nature Publishing Group.

[90] Martijn P. van den Heuvel, René S. Kahn, Joaquín Goñi, and Olaf Sporns. High-cost, high-capacity backbone for global brain communication. Proceedings of the National Academy of Sciences, 109(28):1137211377, July 2012. Publisher: National Academy of Sciences Section: Biological Sciences.

[91] Matthew A. Smith and Adam Kohn. Spatial and Temporal Scales of Neuronal Correlation in Primary Visual Cortex. Journal of Neuroscience, 28(48):12591-12603, November 2008. Publisher: Society for Neuroscience Section: Articles.

[92] Henry Markram, Eilif Muller, Srikanth Ramaswamy, Michael W. Reimann, Marwan Abdellah, Carlos Aguado Sanchez, Anastasia Ailamaki, Lidia Alonso-Nanclares, Nicolas Antille, Selim Arsever, Guy Antoine Atenekeng Kahou, Thomas K. Berger, Ahmet Bilgili, Nenad Buncic, Athanassia Chalimourda, Giuseppe Chindemi, Jean-Denis Courcol, Fabien Delalondre, Vincent Delattre, Shaul Druckmann, Raphael Dumusc, James Dynes, Stefan Eilemann, Eyal Gal, Michael Emiel Gevaert, Jean-Pierre Ghobril, Albert Gidon, Joe W. Graham, Anirudh Gupta, Valentin Haenel, Etay Hay, Thomas Heinis, Juan B. Hernando, Michael Hines, Lida Kanari, Daniel Keller, John Kenyon, Georges Khazen, Yihwa Kim, James G. King, Zoltan Kisvarday, Pramod Kumbhar, Sébastien Lasserre, Jean-Vincent Le Bé, Bruno R. C. Magalhães, Angel Merchán-Pérez, Julie Meystre, Benjamin Roy Morrice, Jeffrey Muller, Alberto Muñoz-Céspedes, Shruti Muralidhar, Keerthan Muthurasa, Daniel Nachbaur, Taylor H. Newton, Max Nolte, Aleksandr Ovcharenko, Juan Palacios, Luis Pastor, Rodrigo Perin, Rajnish Ranjan, Imad Riachi, José-Rodrigo Rodríguez, Juan Luis Riquelme, Christian Rössert, Konstantinos Sfyrakis, Ying Shi, Julian C. Shillcock, Gilad Silberberg, Ricardo Silva, Farhan Tauheed, Martin Telefont, Maria Toledo-Rodriguez, Thomas Tränkler, Werner Van Geit, Jafet Villafranca Díaz, Richard Walker, Yun Wang, Stefano M. Zaninetta, Javier DeFelipe, Sean L. Hill, Idan Segev, and Felix Schürmann. Reconstruction and Simulation of Neocortical Microcircuitry. Cell, 163(2):456-492, October 2015. Publisher: Elsevier.

[93] Stephan Gerhard, Alessandro Daducci, Alia Lemkaddem, Reto Meuli, Jean-Philippe Thiran, and Patric Hagmann. The Connectome Viewer Toolkit: An Open Source Framework to Manage, Analyze, and Visualize Connectomes. Frontiers in Neuroinformatics, 0, 2011. Publisher: Frontiers.

[94] Rodrigo Perin, Thomas K. Berger, and Henry Markram. A synaptic organizing principle for cortical neuronal groups. Proceedings of the National Academy of Sciences, 108(13):5419-5424, March 2011. Publisher: National Academy of Sciences Section: Biological Sciences.

[95] Aaron J. Gutknecht, Michael Wibral, and Abdullah Makkeh. Bits and Pieces: Understanding Information Decomposition from Part-whole Relationships and Formal Logic. arXiv:2008.09535 [cs, math, q-bio], August 2020. arXiv: 2008.09535. 
[96] Thomas Varley and Erik Hoel. Emergence as the conversion of information: A unifying theory. arXiv:2104.13368 [cs, math], April 2021. arXiv: 2104.13368.

\section{$5 \quad$ Materials \& Methods}

\subsection{Basic procedures}

Neural activity was recorded simultaneously from many channels in two female and one male rhesus macaque monkeys (Animals S, Z, and M; body weight 9, 7, and $10 \mathrm{~kg}$, respectively). Detailed experimental procedures have been described previously $[71,17]$. All procedures and animal care were in accordance with German and European law and were in agreement with the Guidelines for the Care and Use of Mammals in Neuroscience and Behavioral Research (National Research Council, 2003)

\subsection{Behavioral Task}

Figure 1 illustrates the time course of the behavioral task described in detail previously [71, 17]. Monkeys were trained to perform a mixed free-choice and instructed delayed grasping task, with the exception of monkey M, which was trained to perform only a instructed delayed grasping task. They were seated in front of a handle that could be grasped in two different ways and visual cues were displayed on a masked monitor that was superimposed on the handle using a beam splitter mirror. Trials started after the monkey placed both hands (for monkey $\mathrm{S}$ ) or one hand (for monkey $\mathrm{Z}$ and $\mathrm{M}$ ) on the resting positions. For monkey $\mathrm{Z}$ and $\mathrm{M}$, the non-task relevant arm was comfortably restrained in an acrylic glass tube during the experiment. Next, a small red disk was displayed centrally on the monitor, which had to be fixated for a variable period of 600 to 1000ms (fixation epoch). After the fixation epoch, cues in the form of larger disks were shown next to the fixation disk for $300 \mathrm{~ms}$ and the handle was illuminated. In the instructed context, one of two discs was displayed instructing the monkey about the required grip type (power or precision; cue epoch). In the free-choice context both disks were displayed indicating the monkey to freely choose between the two grip types. After the cue was turned off, the monkey had to remain steady for a variable time of 1100 to $1500 \mathrm{~ms}$ (memory epoch). The turning off of the central fixation disk instructed the monkey to reach and grasp the target with the required grip type (movement epoch) to receive a liquid reward. Note that to encourage the monkey to perform both grip types during the free-choice context, the reward was iteratively reduced every time the monkey repeatedly chose the same grip type (fraction of power grip selections: $38.5 \pm 4.2 \% \mathrm{SD}$ and $53.3 \pm 5.2 \% \mathrm{SD}$ for $\mathrm{S}$ and $\mathrm{Z}$, respectively; Note that no choice ratio is available for monkey $\mathrm{M}$, since he performed only instructed trails). All trials were randomly interleaved and executed in darkness. Note that the behavioral task also contained delayed instructed trials for monkey S and Z, which were not analyzed in this study. To increase the reliability of the information dynamics estimation, instructed and free choice trials for the same grip type were pooled for all further analyses.

\subsection{Electrode Implantation and Data Recordings}

Surgical procedures have been described in detail previously $[71,17]$. Briefly, two floating microelectrode arrays (FMAs; Microprobes for Life Sciences; 32 electrodes; spacing between electrodes: $400 \mu \mathrm{m}$; length: 1.5 to $7.1 \mathrm{~mm}$ monotonically increasing to target grey matter along the sulcus) were implanted per area in the ventral premotor cortex (area F5), in the anterior intraparietal area (AIP), and in monkey $\mathrm{M}$ also in the 
primary motor cortex (M1) for a total of 128 electrodes for monkey S and Z, and 192 electrodes for monkey $\mathrm{M}$. Monkeys $\mathrm{S}$ and $\mathrm{M}$ were implanted in the left and monkey $\mathrm{Z}$ and the right hemispheres (Figure 1B).

Extracellular signals from the implanted arrays were amplified and digitally stored using a 128-channel recording system for monkey S and Z (Cerebus, Blackrock Microsystems, sampling rate $30 \mathrm{kS} / \mathrm{s}, 0.6-7500 \mathrm{~Hz}$ band-pass hardware filter) and a 256 channel Tucker-Davis system for monkey M (TDT RZ2; sampling rate $24.414 \mathrm{kS} / \mathrm{s}, 0.6-10,000 \mathrm{~Hz}$ band-pass hardware filter) while the monkeys performed the delayed grasping task. All data were saved to disk and analyzed using custom MATLAB code (The Mathworks Inc., Natick, MA).

\subsection{Data Preprocessing}

For spike detection, the broadband signals were first low-pass filtered with a median filter (window length $3 \mathrm{~ms}$ ) and the result subtracted from the raw signal, corresponding to a nonlinear high-pass filter. The signal was then low-pass filtered (4th order non-causal Butterworth filter, fc: $5000 \mathrm{~Hz}$ ). To eliminate common noise sources, principal component (PC) artifact cancellation was applied for all electrodes of each array, as described previously $[72,17]$. To ensure that no true spikes were eliminated from individual channels, PCs with any normalized coefficient greater than 0.36 (conservatively chosen) were retained. Spike waveforms were detected and semi-automatically sorted using a modified version of the offline spike sorter WaveClus $[73,74,17]$.

Units were classified as single- or non-single unit based on five criteria:

1. The presence of short (1-2ms) intervals in the inter-spike interval histogram for single units;

2. The similarity of all waveforms of each unit at each time point;

3. The separation of waveform clusters in the projection of the first 13 features (a combination for optimal discriminability of PCs, single values of the wavelet decomposition, and samples of spike waveforms) detected by WaveClus;

4. The presence of well-known waveform shapes characteristics for single units;

5. The shape of the inter-spike interval distribution.

After the semiautomatic sorting process, redetection of the different average waveforms (templates) was done to detect overlaid waveforms $[75,17]$. To achieve this, filtered signals were convolved with the templates starting with the biggest waveform. Independently for each template, redetection and resorting was run automatically using a 32-dimensional linear discriminate analysis for classification of waveforms. After spike identification, the target template was temporally matched and subtracted from the filtered signal of the corresponding channel to reduce artifacts during the detection of the next template. This procedure allowed us to detect spikes with a temporal overlap up to $0.2 \mathrm{~ms}$. Unit isolation was evaluated again, based on the five criteria mentioned above, to determine the final classification of all units into single or non-single units. Stationarity of firing rate was checked for all units and in case it was not stable over the entire recording session (more than 30\% change in firing rate between the first $10 \mathrm{~min}$ and the last $10 \mathrm{~min}$ of recording) the unit was excluded from further analyses ( $<3 \%$ of all single units). Note that only well isolated single neurons based on the five criteria above were used for all further analysis. 


\subsection{Sliding Window Analysis}

To assess how effective connectivity patterns change with the behavior of the macaque, we made use of a sliding-windows analysis [76]. We used $800 \mathrm{~ms}$ windows, incremented with $100 \mathrm{~ms}$ tied to either the onset of the cue condition (starting with -800ms from the onset of the cue and continuing for $1700 \mathrm{~ms}$ ), or the onset of movement (starting with $-800 \mathrm{~ms}$ from the onset of movement and continuing for $600 \mathrm{~ms}$.) The result was $\approx 900$ individual slices across the 10 different recordings.

For each of these slices, we inferred a single multivariate transfer entropy network using the IDTxl package [25], as well as the local active information storage, and triadic synergies following the pipeline outlined in $[13,14]$.

We chose the sliding window approach to temporal localization instead of the more fine-grained analysis of local information dynamics [77] to ensure that we could see how the overall network topology changed in time: a local information approach would have improved our temporal resolution but at the cost of providing a static network, since fixation, cue, and movement conditions would need to be aggregated to construct a single joint probability distribution (which the system presumably visits different parts of in different conditions, which the local analysis would reveal). This would have precluded a dynamic network approach, which is a core aspect of this study.

\subsection{Active Information Storage}

Active information storage $(A I S)$ is arguably the simplest of the three information dynamics explored here, and quantifies how much information about the next state of the neuron is encoded in the past $k$ states [77]. $A I S$ can be thought of as a measure of the "memory" capacity of the neuron: if every subsequent state is chosen at random, then $A I S=0$, as knowing the past does nothing to reduce our uncertainty about the mediate future, however if the past states of the neuron affects the probability of a particular next state, then the system can be thought of as "remembering" that information and "using" it when deciding what the next state will be (note the scare quotes). A simple example might be the refractory period that follows an action potential: following an action potential, most neurons cannot immediately fire again, as the correct charge needs to be built up. Consequently, an observer attempting to model the dynamics of a single neuron would know that the probability of a spike goes down if the neuron is known to have fired in some previous time-window, and this would be reflected in the AIS measure. For a more involved discussion of AIS in understanding neural dynamics see [4].

The AIS of a single variable $X$ at time $t$ is given as the mutual information between the immediate state of $X$ at time $t$ and the embedded joint-state of the past $k$ time-steps (the embedding here is similar to a Takens embedding [78]):

$$
A I S^{-k}(X)=I\left(X_{t-1}^{-k} ; X_{t}\right)
$$

Intuitively, this can be understood as measuring how much knowledge of the joint past reduces our uncertainty about the immediate future. The free parameter $k$ indicates how many steps into the past we want to consider when calculating the AIS and should be chosen with some care. If $k$ is too small, then we run the risk of missing out on relevant information from more than $k$ steps in the past and we will under-estimate the true $A I S$. On the other hand, if $k$ is too large, then we run the risk of under-sampling the joint-probability space and over-estimating the true $A I S$.

In this work, we set $\max k=3$, which given $5 \mathrm{~ms}$ bins works out to a considered history of $15 \mathrm{~ms}$ (based 
on prior work reported in [79, 80], and then used the IDTxl package [25] to infer an optimal non-uniform embedding. Following [14, 15], the AIS of a neuron was normalized by dividing it by the total Shannon entropy of the neuron. This provides a simple correction for variable firing rates, removing the confound that higher-entropy neurons have a greater capacity for any information dynamic.

\subsection{Multivariate Transfer Entropy Networks}

While the AIS is restricted to understanding the information dynamics of a single neuron (how it is "remembered" through time), the transfer entropy (TE) describes how information "flows" from one source neuron (or a set of source neurons) to a single target neuron [77, 16, 81] For a source $X$ and a target $Y, T E(X \rightarrow Y)$ is given by the mutual information between $X$ 's past and $Y$ 's immediate next state, conditioned on $Y$ 's own past.

$$
T E^{k, l}(X \rightarrow Y)=I\left(X_{-k: t-1} ; Y_{t} \mid Y_{-l: t-1}\right)
$$

By conditioning on $Y$ 's past, we are able to extract the information that $X$ 's past provides about $Y$ 's future above and beyond the AIS in $Y$. Transfer entropy has become an established tool in modeling neural data $[82,5,20,13,46]$. A common technique is to use transfer entropy to construct an effective connectivity graph meant to model the directed synaptic structure of the underlying biological network, to reveal properties like rich clubs [83, 14], multiplex structures [84], and communities [85].

The "classical" transfer entropy has a significant limitation, however that it is strictly bivariate, and consequently blind to higher-order information dynamics. Consider the case of two source neurons $A, B$ synapsing onto a single single target neuron $C$, which implements $C_{t}=X O R\left(A_{t-1}, B_{t-1}\right)$. Due to the synergistic nature of the logical XOR, bivariate transfer entropy will not find any relationship between $A$, $B$ and $C$, since information about $C_{t}$ is only disclosed by the joint pasts of $A$ and $B$ considered together. To address this, we can use the conditional transfer entropy, which quantifies the information flow from one neuron to another in the context of other neurons in the system. Formally:

$$
m T E^{k, l, m}(X \rightarrow Y \mid \mathbf{Z})=I\left(X_{-k: t-1} ; Y_{t} \mid Y_{-l: t-1}, \mathbf{Z}_{-m: t-1}\right)
$$

Where $\mathbf{Z}$ can be a multidimensional variable. Note again that each variable can have a different lag, to account for differences in the intrinsic dynamics in each element. In our simple logical XOR example, $T E(A \rightarrow C)=0$ bit, but $T E(A \rightarrow C \mid B)=1$ bit.

For a would-be network neuroscientist, this presents an optimization problem: for a pair of neurons $X$ and $Y$, how do we find the smallest set of neurons $Z$ such that the context $Z$ provides discloses all the relevant information flow from $X$ to $Y$. The answer is the multivariate transfer entropy $(m T E$ inference, detailed in [23], which uses greedy optimization and multi-level statistical hypothesis testing to define a "parent" set for every neuron in the network, from which the optimal conditional transfer entropy between every parent in the parent set and the target neuron can be derived. Work using simulated data has shown that the bivariate and multivariate transfer entropies return networks with strikingly different topologies and that the multivariate transfer entropy networks are reliably closer to the "ground truth" [24, 86]. It has been established previously that non-trivial synergistic information dynamics exist in biological neural networks $[14,87,15]$, suggesting that bTE, which is known to be blind to synergistic relationships, is missing important aspects of the system's dynamics.

As with the $A I S$, the choice of embedding parameters is important, and to maintain consistency, we again 
set the maximal $k$ value to 3 , and used the IDTxl package [25] to find optimal non-uniform embeddings for each neuron. Due to the large number of neurons recorded, we inferred the parent set of each neuron independently and constructed a putative $m T E$ network post-hoc without correcting for familywise error rates at the whole-network level (every edge was corrected for multiple comparisons). This is an acknowledged limitation of our inference, however the computational requirements were beyond our capabilities at present.

\subsubsection{Density, Rich Club Coefficient \& Clustering Coefficient}

For a directed network $G=(V, E)$, the density of the network is given as:

$$
\frac{|E|}{|V|^{2}-|V|}
$$

Which records the ratio of the number of edges that exist in the network to the total number of edges that could exist after self-loops are removed.

For a directed, weighted graph, the local clustering coefficient for a node $v$ is given by:

$$
c_{v}=\frac{1}{\operatorname{deg}(v)(\operatorname{deg}(v)-1)-2 \operatorname{deg}^{-1}(v)} T(v)
$$

where $\operatorname{deg}(v)$ is the sum of the in- and out-degrees of $v, \operatorname{deg}^{-1}$ is the reciprocal of the degree of $v$, and $T(v)$ counts the number of directed triangles that $v$ participates in. We used the Networkx clustering toolbox [88] for this calculation. The average clustering coefficient for the whole network is given by:

$$
C(G)=\frac{1}{|V|} \sum_{v \in V} c_{v}
$$

Which simply the unweighted average of each local clustering coefficient.

The rich club coefficient quantifies the extent to which nodes of a network with a high number of connections are stronger connected with each other then expected by chance [89, 90]. We calculated a modified version of the original weighted rich club coefficient as described in detail in the following [14]. The weighted rich club coefficient was calculated separately for all effective mTV networks (23 time windows x 2 grasping conditions). First, all neurons per networks were sorted by the combined in and out degree to obtain a degree rank $r_{d e g}$ per neuron. Second, the weighted rich club coefficient $\Phi_{w}\left(r_{d e g}\right)$ was consecutively calculated given by:

$$
\Phi_{w}\left(r_{d e g}\right)=\frac{W_{r_{d e g}}}{r_{d e g}^{2}-r_{d e g}}
$$

with $W_{r_{d e g}}$ denoting the sum of weighted connections of the subset of neurons with a degree rank $>r_{d e g}$, and with $r_{d e g}^{2}-r_{d e g}$ denoting the maximum possible number of connections of the same subset. Since neurons with a high degree have an increased probability by chance to be connected to other neurons with a high degree, the weighted rich club coefficient needs to be normalized by surrogate data based on networks with the same degree and ideally strength distribution. The estimation of the surrogate networks is described in detail below. In a third step, each rich club value was normalized by the corresponding average of 1000 surrogate weighted rich club coefficients. Finally, the normalized weighted rich coefficients were resampled to 1 to 100 using linear interpolation to average across different recording sessions with different numbers of neurons. 


\subsubsection{Surrogate Networks}

To estimate the normalized cluster coefficient, the normalized rich club coefficient and for statistical purposes we generated 1000 partitions of surrogate networks for each inferred multivariate transfer entropy network (21 time windows $\times 2$ conditions $\times 10$ recording sessions). Surrogate networks were generated by first shuffling network connections and second reassigning connection weights. Networks of neurons estimated from simultaneous extracellular recordings are constrained by multiple anatomical and technical factors. To ensure that the above network analyses and statistics are not biased by these factors, network properties affected by these factors should be preserved in the surrogate networks. Several studies have shown, that the number and strength of neuronal connectivity decreases with spatial distance [91, 92, 17]. Additionally, the spatial electrode configuration on each array as well as the configuration of the recording arrays to each other results in a spatial inhomogeneous subsampling of neurons [93, 17]. To account for both, we held the number of connections on the same electrode, the same array, the same cortical area, and the different inter-area connections the same and matched the average connection strength per distance category as close as possible. Cortical neurons also have an increased probability to be reciprocally connected to each other [94]. For this reason, we also held the ratio of reciprocal to unidirectional connections per distance category the same. Furthermore, for the normalization of the rich club coefficient it is necessary that the number of connections per neuron is preserved because strongly connected neurons have an increased probability of being connected to each other by chance $[89,90]$. However, the same is true for the connection strength per neuron, which has been ignored in the literature so far. We therefore not only held the number of connections per neuron the same, but also matched the connection strength per neuron as close as possible. To our knowledge the described above surrogate network method is more conservative than any other method used in the field, which emphasizes the validity of the results based on this surrogate method.

\subsubsection{Cluster-Based Significance Testing}

For the statistical testing of increased rich club and clustering coefficient over the time course of the task, we performed cluster-based surrogate tests. Statistical testing was performed across recording sessions and separate for the power and precision condition (Fig. 1 A) [37, 17]. The testing procedure was the same in both cases and the same set of surrogate networks was used as described above. The computation of the cluster-based surrogate tests was performed as following:

1. Test values (rich club coefficient or rich club coefficient) were calculated separately for all surrogate networks of all time windows and recording sessions.

2. Normalize all surrogate test values by dividing the average surrogate test values of the corresponding time window and recording session (normalized rich club coefficient or normalized rich club coefficient).

3. Average all surrogate test values per partition and time window (for the normalized rich club coefficient the interval between the $83 \mathrm{rd}$ and 95 th percentiles) to obtain one value per network.

4. Calculate the surrogate t-values across recording sessions for all partitions and time windows.

5. Select all surrogate t-values larger than a thresholding criterion and cluster and sum them on the basis of temporal adjacency.

6. Take the largest summed surrogate t-value per partition to construct a distribution of largest summed surrogate t-values. 
7. Repeat step 1-5 for the recorded test values.

8. For every summed t-value calculate the proportion of surrogate $t$-values that are larger than the recorded summed t-values, which corresponds to the p-value.

9. Compare each p-value with a critical alpha-level (0.05 in all cases).

Note that this single comparison replaces the multiple comparisons of the test-values over time.

\section{8 mTE and AIS Distribution Similarity Analysis}

Similarity matrices derived from the time-resolved TE and AIS analysis underwent clustering and community detection to extract, in a purely data-driven way, time periods that had a distinct TE similarity structure. To create similarity matrices for the distribution of AIS values across the nodes, we created a matrix where cell $i j$ gives the cosine similarity between the vector of AIS values across all nodes in the $i^{t h}$ and $j^{\text {th }}$ windows. Cosine similarity is given as:

$$
\operatorname{Sim}(X, Y)=\frac{\langle X, Y\rangle}{\|X\| \times\|Y\|}
$$

Where $\langle X, Y\rangle$ is the dot product that $\|X\|$ is the norm of $\mathrm{X}$. The implementation we used was from the Scikit-Learn Pairwise Comparisons package.

The mTE pipeline was the same, although we took the cosine similarity of the flattened adjacency matrices. First, similarity matrices were aggregated across the 10 trial repetitions by taking the mean. This resulted in a single $46 \times 46$ element matrix for both mTE and AIS. These matrices were then clustered using a version of modularity maximization (employing the Potts null model) and multi-resolution consensus clustering [38]. The resolution parameter, used by the Louvain algorithm, was stepped through a range of 1,000 values covering modular partitions yielding between 2 and 46 modules (the minimal and maximal number of modules possible). The resulting 1,000 partitions were aggregated into a single co-classification matrix which was scaled between followed by subtraction of an analytic null that captures the expected co-classification (mean propensity for each node pair to be grouped in the same community) under random permutation of the module assignments [38]. The resulting scaled co-classification matrices were then subjected to consensus clustering with $\tau=0$. The level of $\tau$ corresponds to the (constant) null. The resulting consensus communities correspond to time steps that are clustered together based on the similarity of their mTE/AIS similarities. Variations in the number of samples (between 100 and 10,000) and range of resolution parameter (2-23; 2-10) had no effect on the cluster boundaries.

\subsection{Synergy \& Partial Information Decomposition}

The final information dynamic is information modification, which describes information that is somehow non-trivially altered by the interaction of two or more incoming "information streams" [77]. Information modification has historically resisted formalization, although one appealing proposal suggests that synergistic information could serve as a useful foundation with which to understanding information modification [26]. Recall that synergistic information is information is inherently multidimensional information that is not trivially reducible to any of its component parts: it represents information that is, in some sense, "greater than the sum of its parts." Since its initial proposal, synergy has been used to great effect as a measure of non-trivial computational processes in neural cultures [14, 87, 15]. 
Calculating the amount of synergy present in dynamic information is a difficult task. The key development by Williams and Beer [33, 34] was that the joint mutual information between a set of variables and a single target could be decomposed into "partial information atoms", which describes the information specifically encoded in a particular combination of source elements and none simpler (for an alternative derivation of the basics of partial information decomposition that leads to the same results, see [95]). For the case of two source variables $X_{1}$ and $X_{2}$ and a target $Y$, this leads to decomposition into redundant, unique, and synergistic partial information atoms:

$$
I\left(X_{1}, X_{2} ; Y\right)=\operatorname{Red}\left(X_{1}, X_{2} ; Y\right)+\operatorname{Unq}\left(X_{1} ; Y \mid X_{2}\right)+\operatorname{Unq}\left(X_{2} ; Y \mid X_{1}\right)+\operatorname{Syn}\left(X_{1}, X_{2} ; Y\right)
$$

Where $\operatorname{Red}()$ indicates the information about $Y$ that can be extracted from both $X_{1}$ or $X_{2}$, Unq() indicates the information that is uniquely disclosed by only $X_{1}$ or $X_{2}$, and $\operatorname{Syn}()$ indicates the information about $Y$ that is disclosed by the joint states of $X_{1}$ and $X_{2}$ together and no simpler combination of elements. In addition to the mutual information between the joint state of $X_{1}$ and $X_{2}$ and $Y$, the bivariate mutual information can be similarly decomposed:

$$
\begin{aligned}
& I\left(X_{1} ; Y\right)=\operatorname{Red}\left(X_{1}, X_{2} ; Y\right)+\operatorname{Unq}\left(X_{1} ; Y \mid X_{2}\right) \\
& I\left(X_{2} ; Y\right)=\operatorname{Red}\left(X_{1}, X_{2} ; Y\right)+\operatorname{Unq}\left(X_{2} ; Y \mid X_{3}\right)
\end{aligned}
$$

The combination of Eqs. 15, 16, and 17 creates a linear system of equations with three equations, three known values, and four unknown values. If it is possible to determine one of these values (the redundant information, unique information, or synergistic information), the rest can be solved with simple algebra. Unfortunately, classical Shannon information theory doesn't provide a natural measure of any of them (note that $I\left(X_{1} ; X_{2}\right) \neq \operatorname{Red}\left(X_{1}, X_{2} ; Y\right)$ and that $I\left(X_{1} ; Y\right) \neq \operatorname{Unq}\left(X_{1} ; Y \mid X_{2}\right)$.

A peculiarity of the PID framework is that, while it provides an elegant structure with which to understand the decomposition of information, it does not specify how exactly to calculate any of the desired values. It is standard to begin with a redundancy function $\operatorname{Red}()$, from which the remaining PI-atoms can be recursively calculated, although the question of exactly what redundancy function to use is a matter of great contention.

\subsubsection{Redundancy / Synergy Bias}

In addition to the notion of synergy-as-information-modification, the partial information decomposition also provides insights into what joint information is redundantly shared between parents [34]. It is known that different systems can be variously dominated by redundant or synergistic information dynamics [51, 96], although this is a very new area of study. Previous work has suggested that different behavioral states may be associated with different distributions of redundant and synergistic information processing [46], and we hypothesized that the fixation/cue states would have a different ratio of synergistic and redundant information dynamics. The idea of an explicitly normalized redundancy/synergy ratio has been proposed in [96], who found that topologically similar systems can nevertheless have strongly different distributions of partial information over the PI lattice. The calculate the redundancy/synergy ratio $(R S R)$, we first normalize the relevant PI-atoms by the overall joint mutual information, which gives us the proportion of the total information that is purely synergistic and the proportion of the total information that is purely redundant: 


$$
\begin{aligned}
\bar{S}\left(X_{1}, X_{2} ; Y\right) & =\frac{\operatorname{Syn}\left(X_{1}, X_{2} ; Y\right)}{I\left(X_{1}, X_{2} ; Y\right.} \\
\bar{R}\left(X_{1}, X_{2} ; Y\right) & =\frac{\operatorname{Red}\left(X_{1}, X_{2} ; Y\right)}{I\left(X_{1}, X_{2}\right) ; Y}
\end{aligned}
$$

The ratio is then:

$$
R S R\left(X_{1}, X_{2} ; Y\right)=\log \left(\frac{\bar{R}}{\bar{S}}\right)
$$

Following the convention established in [51,96], for the log-redundancy/synergy ratio, a value greater than zero indicates a redundancy-dominated dynamic, while a value less than zero indicates a synergy dominated dynamic.

\subsection{Data \& Code Availability Statement}

All scripts used to produce the results described here are available as Supplementary Material, as will all data necessary to replicate the figures and analysis. 